\title{
52. PETROLOGY OF HYDROTHERMALLY METAMORPHOSED SEDIMENTS AT DEEP SEA DRILLING SITE 477, SOUTHERN GUAYMAS BASIN RIFT, GULF OF CALIFORNIA ${ }^{1}$
}

\author{
Kerry Kelts, Eidgenössische Technische Hochschule, Geologisches Institut, Zürich, Switzerland
}

\begin{abstract}
At DSDP Site 477, late Quaternary diatomaceous muds and delta-derived silty-sand turbidites at 2000 meters water depth have been extensively and progressively altered by a deep-seated heat source beneath a sill. Bulk petrologic and microprobe analyses have identified a crudely zoned paragenesis within 260 meters sub-bottom which ranges from unaltered to slightly altered oozes $(0-50 \mathrm{~m})$, anhydrite-dolomite claystones $(105-125 \mathrm{~m})$, illite-chlorite-pyrite claystones (125-140 m), chlorite-pyrite-calcite-carbonaceous claystones with traces of K-feldspar, albite, epidote (140-190 m), and chlorite-epidote-quartz-albite-pyrrhotite-sphene sandstone $(190-260 \mathrm{~m})$. Several petrologic features suggest rapid processes of ocean floor metamorphism: (1) friable and porous textures, (2) abundant relict grains with overgrowths, (3) idiomorphic habits on epidotes, feldspars, and quartz, and (4) a steep gradient in levels of alteration. Many aspects of this hydrothermal assemblage are similar to hydrothermally metamorphosed sandstones of the Cerro Prieto, Mexico, geothermal area.
\end{abstract}

\section{INTRODUCTION}

Hydrothermal deposits have recently been discovered at spreading centers in the Pacific Ocean (Francheteau et al., 1979; Corliss et al., 1979; Spiess et al., 1980) and have been known for some time in deep brine areas of the Red Sea (e.g., Bischoff, 1969). These deposits have been attributed to the interaction of hot sea water with young pillow basalts in a system with relatively little sediment cover. DSDP Leg 64, Site 477, is the first deep sea hole to explore an active hydrothermal regime that is located at a rift center and is interacting with a cover of rapidly deposited hemipelagic sediment.

This chapter briefly reports the results of a petrologic survey of aspects of a limited number of samples. It is based mainly upon Scanning Electron Microscopy (SEM) and electron microprobe analysis. These data are supplemented by some bulk X-ray diffraction analysis and optical microscopy. The goal of the chapter is to provide some constraints on interpretation of the hydrothermal conditions at the Guaymas rifting centers. It confirms many shipboard identifications which suggested a similarity with the geothermal regimes of the Salton Trough area, in particular the Cerro Prieto field of Northern Sonora. The Guaymas deep sea system differs, however, in important aspects from its continental counterpart-it is, for example, without groundwater flow and meteoric waters for recharging. Instead, processes at Site 477 are similar to heating in a pressure cooker, with fluid movement dependent on self-generated hydrothermal convection cells and limited discharge/recharge to/ from the ocean. Such deposits may be an integral part of the evidence for translational margins during the early stages of opening in some oceans. These margins have now become part of a dismembered record in many orogenic belts.

\footnotetext{
${ }^{1}$ Curray, J. R., Moore, D. G., et al., Init. Repts. DSDP, 64: Washington (U.S. Govt. Printing Office).
}

Some cautionary notes are needed. The data herein are incomplete. Although alteration and contact metamorphism were also observed in the immediate vicinity of sills at several sites (see Kastner, this volume, Pt. 2), this paper focuses only on the thick zone of progressive alteration found beneath upper sills in Holes 477 and 477A. The style of alteration indicates large-scale fluid interaction and a very large heat source, probably a magma body, originating not far below the end of the drill string.

\section{SITE AND SETTING}

Site 477 was selected to examine the nature of heat flow anomalies up to $20 \mathrm{HFU}$ (Williams et al., 1979) in the southern Guaymas Basin rift. Details of the drilling and macroscopic lithological descriptions are given in the site chapter, and summarized in Figure 1. Two overlapping holes were drilled at this location, less than a kilometer apart near the eastern edge of the rift wall; they penetrated to only 265 meters sub-bottom. Both encountered a thick sill $(47 \mathrm{~m}$ and $30 \mathrm{~m})$ at 61 and 32 meters sub-bottom, respectively. Although the sills are at different depths, they are thought to be part of the same event (see Site 477 site chapter, this volume, Pt. 1). This sill, although surrounded by a zone characterized by contact metamorphism (Einsele et al., 1980; Kastner, this volume, Pt. 2), is presently cooled; it forms a heat sink, rather than a source, and also a localized lid over the altered sediments below.

Drilling at Site 477A was terminated after we encountered an ominous black scum oozing from fractures in opened cores. This proved to be a high-temperature residue of carbon-particulate rather than the hydrocarbons that we feared. Butyrate core liners showed the effects of incipient melting (melting temperature $80^{\circ} \mathrm{C}$ ) in spite of the circulating coolant, and the temperature log instrument had to be pulled before equilibrium was established in order to protect the associated gamma log instrument from heat damage at $130^{\circ} \mathrm{C}$. 


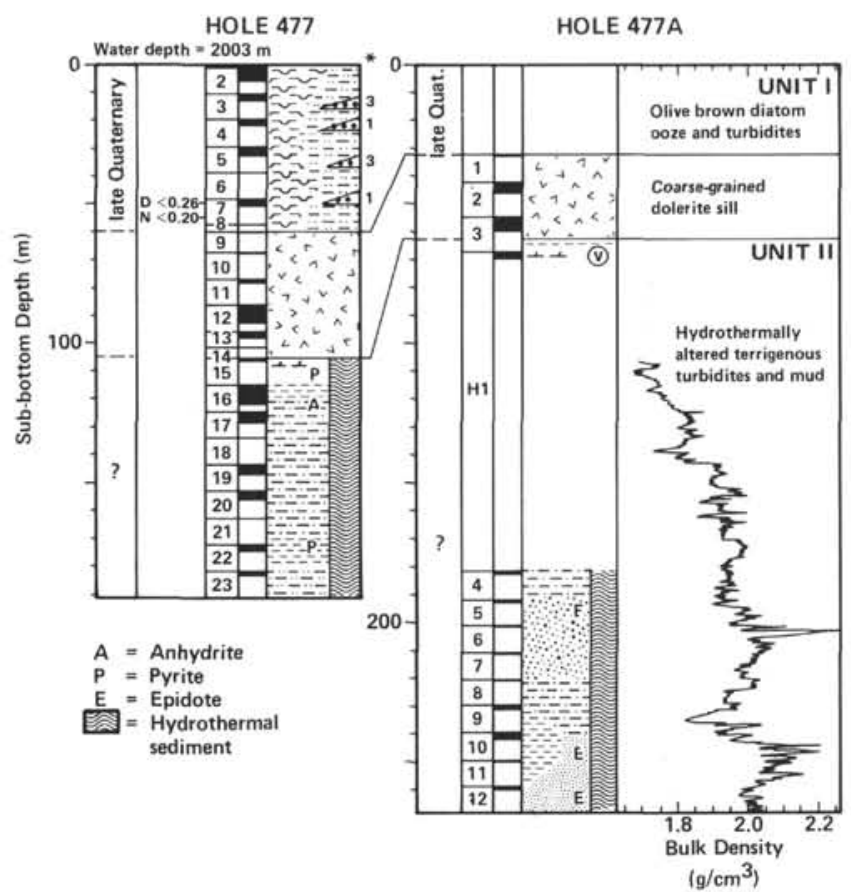

Figure 1. Stratigraphic column for Site 477, with zones of characteristic hydrothermal mineral assemblages.

Poor recovery causes serious difficulties for any systematic petrologic analysis. Above the sill, the section comprises mainly olive drab diatomaceous mud, mostly redeposited. There are scattered intercalations of decimeter-thick, gray, graded silty-sands which are highly disturbed in cores. Many core sections are brecciated, including lithified sediment just above the sill and, below the sill, a series of soft to hard, competent but friable sandstone beds alternating with brittle shales. Below 190 meters, recovery consisted of only a few sandstone fragments for each 9 meters cored. The disappointing recovery does indicate however, that the section is not uniformly lithified, and that soft or brittle layers are preferentially flushed out. We surmise that, before hydrothermal alteration, the section below the sill was a sequence of interbedded diatomaceous muds and turbidites similar to the hydraulically piston-cored Site 481 section of the same age (latest Quaternary) encountered in the northern Guaymas Rift (see Site 481 site chapter, this volume, Pt. 1). The porous, light gray sandstone fragments below 190 meters thus would represent basal parts of the delta-derived turbidites (Einsele and Kelts, this volume, Pt. 2). Consistent with this is the occurrence of relict primary sedimentary structures which include current laminations.

Some idea of the lithology of the missing section can be gleaned from logging results. For example, gamma ray blips at 150 meters in Hole 477 and in a broader zone from 110-140 meters in Hole 477A may correspond to levels containing the highest concentration of potassium-bearing minerals (K-feldspar, illite). Bulk density and sonic velocity logs show various small, sharp peaks at levels of enhanced lithification, possibly in relation to cemented basal turbidite sands. The poor recovery leaves us uncertain about the variability of hydrothermal mineralogy at given levels and their relation to the permeability of each lithology. For example, light gray to dark gray colors are determined by the entrapment of fine carbon-particulate disseminated in less permeable zones.

\section{METHODOLOGY AND DATA}

Shipboard descriptions of smear slides and thin sections are given in the Site 477 site chapter, this volume, Pt. 1. Further shore-based investigation based on thin sections from microprobe mounts (Fig. 2) and smear-slides of samples for scanning electron microscopy (SEM) surveys (Plates 1-4) confirm the general trends reported in the site chapter. Qualitative X-ray diffraction mineralogy was determined for a limited downhole series of bulk, ground powder samples and the results are summarized in Table 1 . Numerous small accessory peaks occur in samples from hydrothermally altered sediments below the sill. Future studies require partitioning and concentration of various mineral fractions for positive identification. Because of the unusual mixtures, I have opted here merely to report original major peak heights in millimeters as a qualitative comparison among samples run with the same settings. Unidentified, conspicuous peaks are reported in angstroms with corresponding peak height in millimeters. A few samples were ultrasonically dispersed and oriented glass mounts prepared for the clay fraction (Fig. 3).

\section{MICROPROBE EXAMINATION}

Seven samples were selected from Araldite-impregnated chips for analysis on an ARL-SEQM microprobe at ETH-Zürich. These are supplemented by sets of microprobe analyses of one sample each from the University of Basel (Operator R. Oberhänsli; 477A-11-1, 11-15 $\mathrm{cm}$ ) and Stanford University (Operator M. Lyle; 477A-5-1, 0-5 cm). The results are tabulated in Table 2. Most other samples above Core 477-23 are too fine-grained to permit accurate positioning of the beam in order to avoid contamination by araldite matrix or adjoining minerals.

The ETH-Zürich instrument has an on-line ZAF correction by program ComicFD Nr. 5 including Bence/Albee approximations (J. Sommerauer, pers. comm., 1979). Acceleration voltage was $15 \mathrm{kV}$ with 20-ma reference current on a brass knob. I measured 10 elements $(\mathrm{Cr}, \mathrm{Ti}, \mathrm{K}, \mathrm{Al}, \mathrm{Na}, \mathrm{Fe}, \mathrm{Si}, \mathrm{Mg}, \mathrm{Ca}, \mathrm{Mn}$ ); thus $\mathrm{Cu}, \mathrm{Zn}$, or other metallic elements possibly occurring in opaque minerals have been overlooked. Standardization every $4 \mathrm{hr}$. showed extremely stable conditions over 2 days. Raw data from epidote and plagioclase were further corrected for absorption, fluorescence, backscatter, and ionization by an EMMA program of the ETH petrography institute.

Stanford analyses (M. Lyle, pers. comm.) also include corrections for fluorescence by continuum and characteristic radiation, mass absorption coefficients, and excitation parameters. Results from the Basel ARL instrument are reported as a first approximation using a 15 $\mathrm{kV}$ beam with ZAF and drift correction.

\section{DISCUSSION}

\section{Unaltered Sediments}

One problem in determining the extent of alteration below the sill is the composition of the original sediment. Because of high sedimentation rates for diatomaceous muds within the rift (over $1200 \mathrm{~m} / \mathrm{m} . \mathrm{y}$.) and a common occurrence of quartz-rich feldspathic sands above the sill, it seems reasonable to assume that the altered sequence consisted of the same anoxic muds, rich in organic carbon $\left(2-4 \% \mathrm{C}_{\mathrm{org}}\right)$ and with an approximate bulk composition of $40 \%$ diatomaceous opal and $40 \%$ terrigenous clastics. Approximately $5-10 \%$ carbonate occurs as scattered benthic and planktic foraminifers and nannofossils, together with some scattered hash from benthic mollusks. A survey of the composition of river detritus (Aguayo, this volume, Pt. 2) shows that the Yaqui River system (Sonora, Mexico) is quartz-dom- 


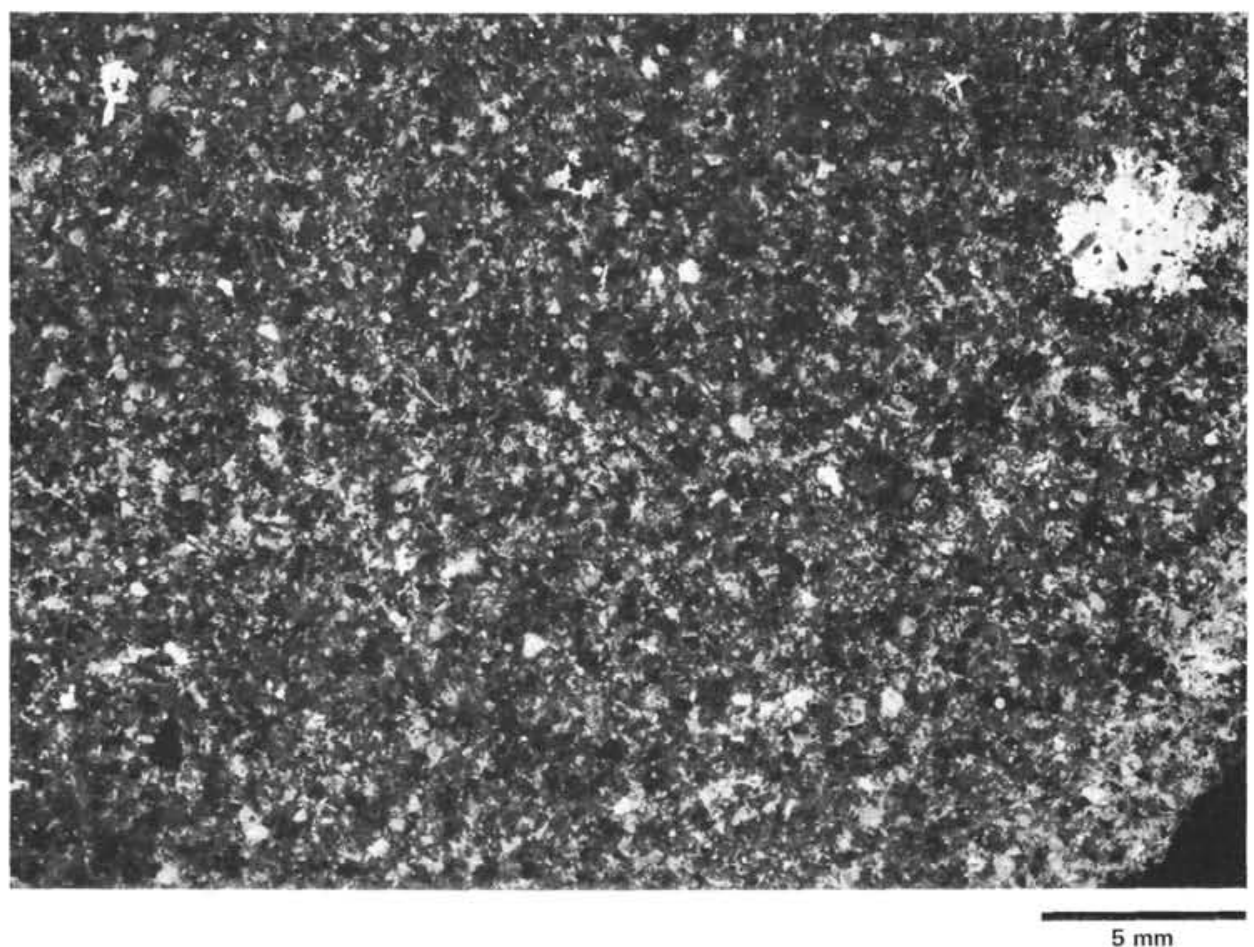

Figure 2. Obliquely reflected light microphotograph of a microprobe thin section (Sample 477A-11-1, $15-17 \mathrm{~cm}$ ). Fine-grained, equigranular quartz(dark!)-plagioclase sandstone with scattered idiomorphic epidote crystals and numerous $0.1-5-\mathrm{mm}$ patches of subhedral pyrrhotite (bright white) as intergranular fill (see Plates 1 and 4).

Table 1. Results of bulk X-ray diffraction mineralogy (XRD).

\begin{tabular}{|c|c|c|c|}
\hline $\begin{array}{l}\text { Sub-bottom } \\
\text { Depth } \\
(\mathrm{m})\end{array}$ & Sample & Description & XRD Results ${ }^{\mathrm{a}}$ \\
\hline \multicolumn{4}{|l|}{ Hole 477} \\
\hline & $4-1,63-65 \mathrm{~cm}$ & $\begin{array}{l}\text { Olive brown muddy } \\
\text { diatomaceous ooze }\end{array}$ & $\begin{array}{l}\mathrm{Q}(67), \mathrm{CC}(52, \text { stoichiometric), } \mathrm{Plg}(46,3.21 \AA \AA), \mathrm{KF} \text { (11), clay (broad, minor), } \\
\text { opal (10) }\end{array}$ \\
\hline 32.6 & $5, \mathrm{CC}$ & $\begin{array}{l}\text { Olive brown muddy } \\
\text { diatomaceous ooze }\end{array}$ & $\begin{array}{l}\mathrm{Q}(65), \mathrm{CC}(47), \mathrm{Plg}(52,3.17 \AA), \mathrm{Plg}(39,3.20 \AA), \mathrm{KF}(3), \operatorname{Dol}(28,2.896 \AA) \\
\text { Pyr (4), opal (10), clay (broad, minor) }\end{array}$ \\
\hline \multirow[t]{2}{*}{50.5} & $7-1,40-41 \mathrm{~cm}$ & Hard gray sandstone & $\begin{array}{l}\text { Q (230), Plg (160, mixed), KF (30), Clp (14), Zeol } 2(8,9.42 \AA) \text {, Gyp (17), } \\
\text { Pyr (20), Epi (traces?) }\end{array}$ \\
\hline & $15-1,61 \mathrm{~cm}$ & $\begin{array}{l}\text { Anhydrite clusters } \\
\text { and gray mudstone }\end{array}$ & $\begin{array}{l}\mathrm{Q}(208) \text {, Anh (116), Plg (10, mixed), Dol }(16,2.90 \AA) \text {, Gyp (12), clay }(12.5-15 \AA \text {, } \\
\text { broad), other minor }\end{array}$ \\
\hline 118 & $16-3,42-44 \mathrm{~cm}$ & $\begin{array}{l}\text { Fine grained med. } \\
\text { gray mudstone }\end{array}$ & $\begin{array}{l}\text { Q (230), Anh (66), Plg }(18,3.20 \AA), \operatorname{KF}(22,3.24 \AA), \mathrm{Chl}(14), \mathrm{ll}(13), \mathrm{CC}(4) \text {, } \\
\text { Pyr (7), Gyp (trace) }\end{array}$ \\
\hline \multirow[t]{3}{*}{124} & $\begin{array}{l}17-1,127 \mathrm{~cm} \\
17-2,28 \mathrm{~cm}\end{array}$ & $\begin{array}{l}\text { Soft brown clay } \\
\text { Lt. gray mudstone }\end{array}$ & $\begin{array}{l}\mathrm{Q}(230) \text {, Anh (116), Plg }(56,3.20 \AA), \mathrm{Chl}(21), \mathrm{ml}(10), \mathrm{Pyr}(8), \mathrm{KF}(12) \\
\mathrm{Q}(220) \text {, Anh }(230), \mathrm{Plg}(34), \mathrm{KF}(16), \mathrm{Dol}(17,2.86 \AA), \mathrm{CC}(6), \text { Gyp ( } 45) \text {, } \\
\text { Chl (11), III (5), other }(3.07 \AA)\end{array}$ \\
\hline & $19-1,20 \mathrm{~cm}$ & $\begin{array}{l}\text { Lt. brown dolostone } \\
\text { (downhole caving?) }\end{array}$ & $\mathrm{Q}(23), \mathrm{Dol}(230,2.884 \AA), C C(10,3.018 \AA)$ \\
\hline & $19-1,67 \mathrm{~cm}$ & $\begin{array}{l}\text { L.. gray sandy } \\
\text { mudstone }\end{array}$ & $\begin{array}{l}\mathrm{Q}(230), \mathrm{Plg}(114,3.20 \AA), \mathrm{CC}(123), \mathrm{KF}(25), \text { III }(11,9.85 \AA), \mathrm{Chl}(34) \text {, other } \\
(2.99 \AA),(21,2.91 \AA),(6,2.82 \AA),(2,1.80 \AA),(14,6.38 \AA)\end{array}$ \\
\hline \multirow[t]{2}{*}{156} & $20-2,14 \mathrm{~cm}$ & Dark gray mudstone & $\begin{array}{l}\mathrm{Q}(230), \mathrm{Plg}(76,3.21 \AA), \mathrm{Chl}(37,7.15 \AA), \text { III }(10), \mathrm{KF}(20), \operatorname{Pyr}(9), \mathrm{CC}(9) \text {, } \\
\text { other }(8,6.4 \AA),(13,4.72 \AA),(13,2.91 \AA),(17,3.00 \AA),(11,2.826 \AA)\end{array}$ \\
\hline & $21-1,26 \mathrm{~cm}$ & Dark gray mudstone & $\begin{array}{l}\mathrm{Q}(222), \operatorname{Plg}(131,3.20 \AA), \mathrm{Chl}(38), \mathrm{Pyr}(18), \text { III }(10), \mathrm{KF}(16), \mathrm{CC}(4) \text {, other } \\
(13,2.99 \AA),(6,2.912 \AA)\end{array}$ \\
\hline 173 & $22-1,76 \mathrm{~cm}$ & $\begin{array}{l}\text { Dark gray pyritic } \\
\text { mudstone }\end{array}$ & $\begin{array}{l}\mathrm{Q}(225), \mathrm{Plg}(104), \mathrm{Chl}(32), \mathrm{KF}(20), \operatorname{Pyr}(12), \mathrm{CC}(14), \text { other }(34,2.70 \AA) \\
(10,3.30 \AA),(47,2.21 \AA)\end{array}$ \\
\hline 182 & $23-1,19 \mathrm{~cm}$ & Dark gray mudstone & $\mathrm{Q}(230), \mathrm{Plg}(95), \mathrm{Chl}(30), \mathrm{KF}(15), \mathrm{CC}(13), \mathrm{Pyr}(3)$, other $(8,2.70 \AA)$ \\
\hline \multicolumn{4}{|l|}{ Hole 477A } \\
\hline & $5-1,21 \mathrm{~cm}$ & $\begin{array}{l}\text { Lt. gray friable } \\
\text { sandstone }\end{array}$ & $\mathrm{Q}(230), \mathrm{Plg}(225, \mathrm{Ab} 80), \mathrm{Chl}(58)$, Epi $(16,5.08 \AA)$ \\
\hline & $5-1,84 \mathrm{~cm}$ & $\begin{array}{l}\text { Lt gray, black- } \\
\text { speckled sandstone }\end{array}$ & $\begin{array}{l}\text { Q (240), Plg }(101,3.21 \AA) \text {, Chl }(42 \text {, asymmetry, } 7.10 \AA) \text {, Epi }(33,5.08 \AA) \text {. } \\
\text { Pyr (6), other }(22,3.99 \AA)\end{array}$ \\
\hline & $7, \mathrm{CC}$ & Lt. gray siltstone & $\begin{array}{l}\mathrm{Q}(230), \mathrm{Plg}(148), \mathrm{Chl}(47), \text { Epi }(6) \text {, other }(35,2.527 \AA \text { complex), }(16,2.90 \AA \text {, } \\
2.94 \AA)\end{array}$ \\
\hline 230 & $9-1,73 \mathrm{~cm}$ & $\begin{array}{l}\text { Friable, lt. gray } \\
\text { sandstone }\end{array}$ & $\begin{array}{l}\mathrm{Q}(230), \mathrm{Plg}(136, \mathrm{Ab} 70), \mathrm{Chl}(34), \text { Epi }(13), \mathrm{CC}(4,3.025 \AA), \mathrm{Pyr}(4), \text { new } \\
(2.945 \AA, 2.92 \AA, 2.848 \AA, 2.82 \AA-\text { all about } 10 \mathrm{~mm})\end{array}$ \\
\hline 239 & $10-1,28 \mathrm{~cm}$ & $\begin{array}{l}\text { Fine-grained, soft } \\
\text { sandstone }\end{array}$ & Q (240), Plg (72), Chl (56), Epi (14), Pyr (12), Pyrrh (28, $3.13 \AA$ A), (15, $270 \AA)$ \\
\hline 248 & $11-1,15 \mathrm{~cm}$ & $\begin{array}{l}\text { Hard gray sandstone } \\
\text { (SEM and thin sect.) }\end{array}$ & $\begin{array}{l}\mathrm{Q}(240) \text {, Plg }(154, \text { Ab60). Pyrrh }(38,3.13 \AA \text { Ả), Pyr (12), Epi (7), new (clay } \\
12.8-14.2 \AA, 12, \text { broad) }\end{array}$ \\
\hline
\end{tabular}

Note: Q. quartz; CC, calcium carbonate; Plg, plagioclase; Chl, chlorite; Epi, epidote; KF, K-feldspar; Dol, dolomite; m, illite, 10.0 Aं; Gyp,

gypsum; Anh, anhydrite; Clp, clinotilolite; Zeol, other zeolite; Pyr, pyrite; Pyrrh, pyrrhotite.
Height of major peak reflection given in parentheses in $\mathrm{mm}$, with spacing in angstroms ( $\mathrm{A})$. 


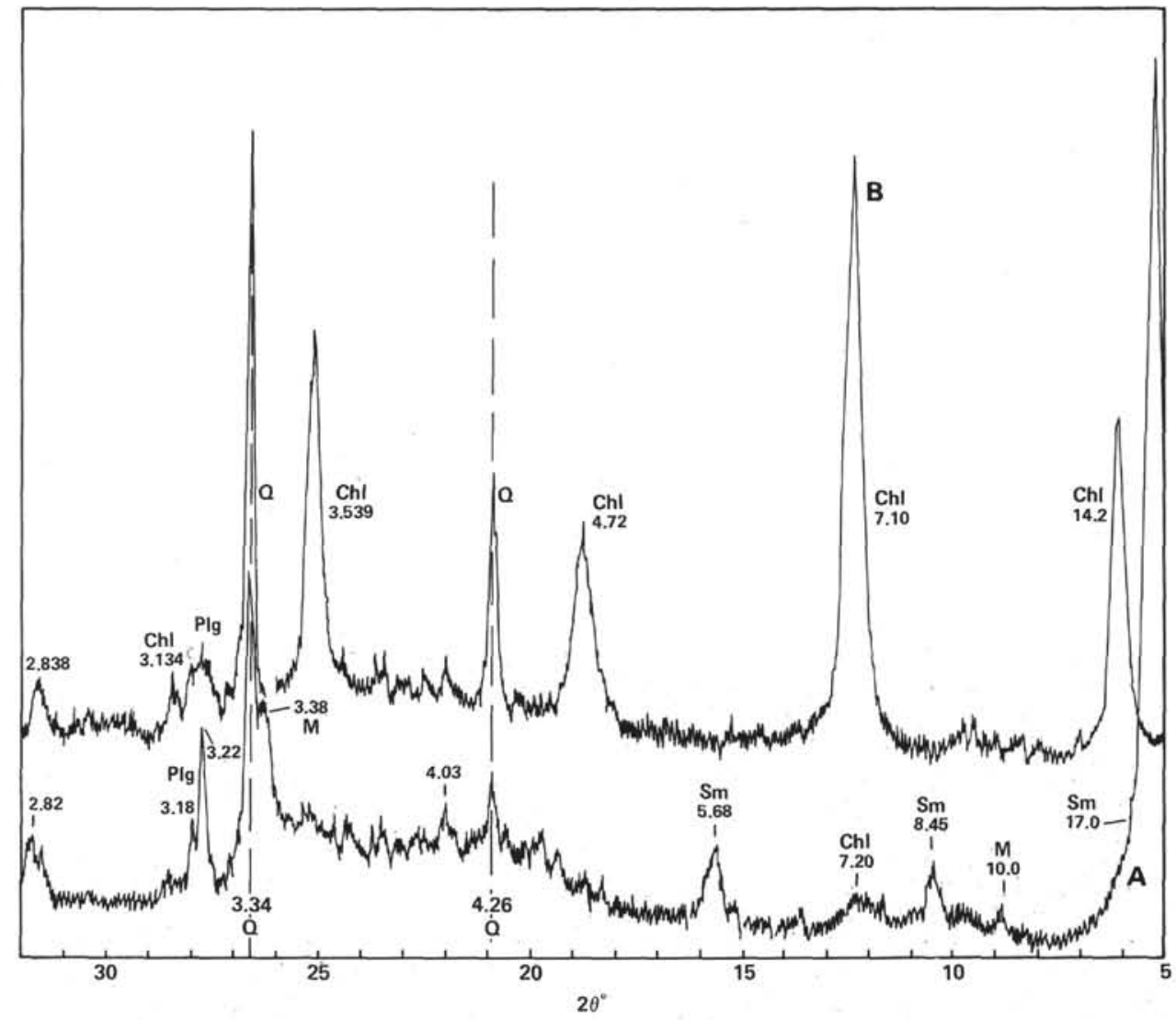

Figure 3. X-ray diffraction diagrams from oriented glass mounts comparing the clay fraction of (A) smectite-illite-rich sandy muds from 12 meters above the upper sill (Sample 477-7-2, $54 \mathrm{~cm}$ ) with (B) two chlorite-rich samples from the middle and lower part of hydrothermally altered claystones to sandstones (Samples 477-23-1, 19-21 cm, at $130 \mathrm{~m}$ and 477A-10-1, $28 \mathrm{~cm}$, at $240 \mathrm{~m}$ ). Abbreviations: Q, quartz, Chl, chlorite, Sm, smectite, M, mica-illite, $\mathrm{Plg}$, plagioclase, $d$-spacing given in angstroms $(\AA)$.

inated but also transports an abundant suite of immature polygenetic feldspars from various volcanic terrains. Rock fragments and mafic minerals are common but carbonate is almost absent. Other sands from along the margin, such as those represented in slope Site 479 , consist of felsic sands with fresh-looking plagioclase grains, which are of broadly median composition and are more abundant than quartz.

Basinal sands from other sites were found to reflect a spectrum similar to the Yaqui system. At nearby Site 478 primary textures are preserved in an unaltered sand sample which has been cemented by early diagenetic dolomite and serves as a reference sample for optical study. Thin sections of this sample show many fresh plagioclase grains with well-developed twinning, clear angular quartz grains, and scattered $\mathrm{K}$-feldspar grains, together with basaltic rock fragments and accessory detrital sphene, ilmenite, and pyroxene. Plagioclase compositions are typically $\mathrm{Ab}_{40-60}$.

Sand grains sieved from Core 477-7, 12 meters above the sill, apparently show some effects of alteration associated with either the contact metamorphic zone of the sill or low-temperature hydrothermal effects. SEM microtextures (Plate 2) indicate some incipient surface breakdown of cloudy plagioclase and a well-bonded layer of smectite clays adhering to some grains. One frag- ment of gray sandstone removed from this level contains traces of two zeolites and some epidote (Table 1, Sample $477-7-1,40 \mathrm{~cm}$ ). This pebble has probably been redeposited from an older scarp with outcropping hydrothermal deposits and emphasizes the problem of basinal reworking of sediments.

\section{Altered Sediment}

\section{Quartz-Silica}

All vestiges of diatom frustules (opal) disappear below the sills. The increased relative abundance of quartz in X-ray patterns (Table 2) suggests that a large portion of the opal has recrystallized as quartz. No opal-CT phases were detected. Much of the silica may have been transported by convecting hot pore waters out of the system. Thin sections of coarser-grained samples show the presence of common quartz relict grains with yellow cathode luminescence and common wavy extinction. A large portion of quartz seems also to occur as tiny (4-10 $\mu \mathrm{m})$ euhedrally terminated prisms forming part of the microlitic replacement of other grains (see Plates 3 and 4). Overgrowths are also common and display sawtooth edges with a crown of small pyramids. Quartz cement is comparatively rare, but in a few instances it is observed as a late-stage infilling, with a mosaic extinction pattern 
Table 2. Results of microprobe analysis.

\begin{tabular}{|c|c|c|c|c|c|c|c|c|c|c|c|}
\hline \multirow{2}{*}{$\begin{array}{l}\text { Measurement } \\
\text { Description }\end{array}$} & \multirow[b]{2}{*}{ Interpretation } & \multicolumn{10}{|c|}{ Composition $(\%)$} \\
\hline & & $\mathrm{SiO}_{2}$ & $\mathrm{Al}_{2} \mathrm{O}_{3}$ & $\mathrm{FeO}$ & $\mathrm{MnO}$ & $\mathrm{CaO}$ & $\mathrm{Na}_{2} \mathrm{O}$ & $\mathrm{K}_{2} \mathrm{O}$ & $\mathrm{TiO}_{2}$ & $\mathrm{MgO}$ & Total \\
\hline \multicolumn{12}{|l|}{$477-17-3,83-87 \mathrm{~cm}$} \\
\hline \multirow{2}{*}{$\begin{array}{l}\text { 1. High RI, bf; needle } \\
\text { 2. Euhedral prism, } \\
\text { small }(10-15 \mu \mathrm{m})\end{array}$} & \multirow{2}{*}{$\begin{array}{l}\text { Mica } \\
\text { Albite (Ab95 +) }\end{array}$} & \multirow{2}{*}{$\begin{array}{l}47.6 \\
67.2\end{array}$} & \multirow{2}{*}{$\begin{array}{l}32.9 \\
19.4\end{array}$} & \multirow{2}{*}{$\begin{array}{l}1.7 \\
0.2\end{array}$} & 0.04 & 0.04 & 0.6 & 8.7 & 0.4 & 0.9 & 92.7 \\
\hline & & & & & & 1.0 & 7.1 & 0.8 & & 0.1 & $95.8^{\mathrm{a}}$ \\
\hline 3. Overgrowth & K-feldspar (pure) & 71.4 & 17.4 & 0.1 & & & & & & & \\
\hline $\begin{array}{l}\text { 4. Euhedral, prism } \\
\text { pseudomorph }\end{array}$ & K-feldspar (pure) & 66.2 & 18.2 & 0.2 & 0.1 & & $\begin{array}{l}0.2 \\
0.7\end{array}$ & $\begin{array}{l}15.9 \\
15.4\end{array}$ & $\begin{array}{l}0.1 \\
0.1\end{array}$ & & $\begin{array}{l}105 \\
100.8\end{array}$ \\
\hline $\begin{array}{l}\text { 5. Euhedral, clear } \\
\text { yellow } \mathrm{CL}\end{array}$ & Quartz & 115 & & & & & & & & & \\
\hline $\begin{array}{l}\text { 6. Small terminated } \\
\text { prisms, yellow } \mathrm{CL}\end{array}$ & Quartz & 114 & & & & & & & & & \\
\hline $477 \mathrm{~A}-6, \mathrm{CC}$ & & & & & & & & & & & \\
\hline 1. Euhedral prism & Quartz & 115 & & & & & & & & & \\
\hline 2. Altered plagioclase & $\mathrm{Ab}_{72}$ & 56.9 & 24.9 & 0.2 & & 8.2 & 6.9 & 0.2 & 0.1 & & $97.3^{\mathrm{a}}$ \\
\hline 3. Prism, matrix & Zeolite & 91.8 & 5.3 & 0.1 & & 1.3 & 3.4 & 0.2 & 0.1 & 0.1 & 102.3 \\
\hline $477 \mathrm{~A}-8, \mathrm{CC}$ & & & & & & & & & & & \\
\hline $\begin{array}{l}\text { 1. Small, euhedral } \\
\text { pinacoid }\end{array}$ & Epidote & 38.4 & 25.8 & 7.9 & 0.2 & 23.7 & & & 0.3 & 0.1 & $96.6^{\mathrm{a}}$ \\
\hline $\begin{array}{l}\text { 2. Long prism in } \\
\text { altered grain }\end{array}$ & Epidote & 38.0 & 23.7 & 8.7 & 0.7 & 23.2 & & & 0.2 & 0.2 & $94.8^{\mathrm{a}}$ \\
\hline $\begin{array}{l}\text { 3. Euhedral edge on } \\
\text { plagioclase }\end{array}$ & Epidote & 38.6 & 25.9 & 7,4 & 0.2 & 23.9 & & & 0.1 & 0.1 & $96.3^{\mathrm{a}}$ \\
\hline $\begin{array}{l}\text { 4. Low bf, euhedral } \\
\text { edge }\end{array}$ & Albite & 58.8 & 20.8 & 0.1 & & 3.8 & 7.1 & 0.1 & & & 90.1 \\
\hline $\begin{array}{l}\text { 5. Large, terminated } \\
\text { prism }\end{array}$ & Epidote & 38.8 & 23.7 & 9.8 & 0.5 & 23.5 & & & 0.2 & 0.2 & $96.7^{a}$ \\
\hline 6. Ore without $\mathrm{Fe}$ & $\mathrm{Cu} ?$ & & & & & & & & & & \\
\hline $\begin{array}{l}\text { 7. Thin prism in } \\
\text { matrix }\end{array}$ & Epidote & 38.2 & 25.1 & 8.7 & 0.3 & 23.6 & & & 0.1 & 0.1 & $96.2^{\mathrm{a}}$ \\
\hline $477 \mathrm{~A}-9-1,80-85 \mathrm{~cm}$ & & & & & & & & & & & \\
\hline 1. Large, opaque & Pyrrhotite & & & 82.1 & & & & & & & \\
\hline 2. Opaque, dull luster & Pyrrhotite & & & 83.1 & & & & & & & \\
\hline $\begin{array}{l}\text { 3. Euhedral in ore } \\
\text { mass }\end{array}$ & Albite Ab93 & 67.7 & 20.3 & 0.3 & & 1.2 & 7.4 & 0.2 & & & $97.4^{\mathrm{a}}$ \\
\hline $\begin{array}{l}\text { 4. Euhedral, large, } \\
\text { blocky }\end{array}$ & Quartz & 114 & & & & & & & & & \\
\hline 5. Large spray, point & Epidote & 39.0 & 27.2 & 6.4 & 0.4 & 24.1 & & & 0.1 & 0.1 & $97.4^{\mathrm{a}}$ \\
\hline 6. End prism & Epidote & 38.9 & 26.7 & 7.1 & 0.8 & 23.7 & & & 0.2 & 0.2 & $97.6^{\mathrm{a}}$ \\
\hline 7. Small, high bf & Epidote & 39.1 & 24.4 & 9.1 & 0.6 & 23.6 & & & 0.4 & 0.2 & $97.6^{\mathrm{a}}$ \\
\hline $\begin{array}{l}\text { 8. Prism in fibrous } \\
\text { matrix }\end{array}$ & Epidote & 38.9 & 24.2 & 10.2 & 0.5 & 23.2 & & & 0.2 & 0.1 & $97.3^{\mathrm{a}}$ \\
\hline $\begin{array}{l}\text { 9. Tiny thomb, high } \\
\text { relief }\end{array}$ & Epidote & 37.8 & 25.1 & 7.1 & 0.6 & 22.6 & 0.1 & & 0.2 & 0.2 & $93.8^{\mathrm{a}}$ \\
\hline $\begin{array}{l}\text { 10. Small flake, low } \\
\text { relief }\end{array}$ & Epidote & 39.3 & 22.8 & 8.9 & 0.4 & 23.6 & & & 0.2 & 0.3 & 95.4 \\
\hline 11. Euhedral & Epidote & 39.1 & 25.0 & 8.6 & 0.7 & 23.3 & & & 0.2 & 0.3 & 97.4 \\
\hline 12. Rim on plagioclase & Chlorite & 39.4 & 13.8 & 7.5 & 0.3 & 1.1 & 4.5 & 0.1 & & 13.0 & 79.5 \\
\hline $\begin{array}{l}\text { 13. Center, altered } \\
\text { plag. }\end{array}$ & Chlorite & 27.1 & 13.1 & 12.8 & 0.5 & 0.1 & 0.5 & & & 19.5 & 73.6 \\
\hline 14. Thin euhedral lath & Albite Ab98 & 72.6 & 18.8 & 0.1 & & 0.3 & 9.6 & 0.4 & & & 101.8 \\
\hline 15. Small grain & Epidote & 38.5 & 24.4 & 9.4 & 0.4 & 23.7 & & & 0.2 & 0.3 & $96.9^{\mathrm{a}}$ \\
\hline $\begin{array}{l}\text { 16. Large subhedral } \\
\text { yellow CL }\end{array}$ & Quartz & 113 & & & & & & & & & \\
\hline 477A-10,CC, No. 1 & & & & & & & & & & & \\
\hline 1. Small prism & Epidote & 38.2 & 21.9 & 12.0 & 0.2 & 22.4 & & & 0.4 & 0.1 & $95.1^{\mathrm{a}}$ \\
\hline 2. Small, stubby & Epidote & 39.4 & 25.4 & 8.9 & 0.5 & 23.6 & & & 0.1 & 0.1 & $98.0^{\mathrm{a}}$ \\
\hline 3. Cluster, small & Epidote & 39.6 & 25.4 & 8.5 & 0.3 & 23.4 & & & 0.2 & 0.1 & $97.5^{\mathrm{a}}$ \\
\hline $\begin{array}{l}\text { 4. Small, high of } \\
\text { cluster }\end{array}$ & Epidote & 39.1 & 24,1 & 9.8 & 0.5 & 23.7 & & & 0.1 & 0.2 & $97.7^{\mathrm{a}}$ \\
\hline 5. Rim on plagiociase & Quartz & 113 & & & & & & & & & \\
\hline $\begin{array}{l}\text { 6. Rim on plag. lath, } \\
\text { low } \mathrm{CL}\end{array}$ & Albite & 70.1 & 19.0 & & & 0.1 & 7.4 & & & & $96.5^{\mathrm{a}}$ \\
\hline $\begin{array}{l}\text { 7. Large pyramid, } \\
\text { cleavage }\end{array}$ & Epidote & 38.8 & 25.1 & 8.0 & 0.3 & 23.5 & & 0. & 0.2 & 0.1 & $96.0^{\mathrm{a}}$ \\
\hline $\begin{array}{l}\text { 8. Double pyramid in } \\
\text { ore }\end{array}$ & Sphene & 26.5 & 3.6 & 0.6 & 0.1 & 24.3 & & 0.1 & 29.0 & 0.2 & 84.4 \\
\hline 9. Small blocky prism & Epidote & 39.3 & 22.0 & 11.5 & 0.4 & 23.0 & & & 0.3 & 0.4 & $96.9^{a}$ \\
\hline 10. Aggregate, matrix & Chlorite & 25.1 & 15.2 & 19.4 & 0.9 & 0.1 & & & 0.1 & 16.7 & 77.5 \\
\hline 11. Double prism, small & Sphene & 32.8 & 0.9 & 1.6 & 0.3 & 28.5 & 0.1 & & 36.0 & & 100.3 \\
\hline 12. Doyble terminated & Epidote & 39.5 & 25.4 & 8.3 & 0.3 & 23.8 & & & 0.2 & 0.1 & 97.7 \\
\hline $\begin{array}{l}\text { 13. Hexagonal plate } \\
\text { faint yellow } \mathrm{CL}\end{array}$ & Plag. $\mathrm{Ab}_{80}$ & 63.6 & 22.3 & 0.5 & & 4.3 & 6.5 & & 0.2 & 0.4 & $97.9^{\mathrm{a}}$ \\
\hline 14. Euhedral, pale $\mathrm{CL}$ & ?Zeolite & 74.9 & 10.6 & 4.3 & 0.2 & 12.9 & & & 0.1 & 0.1 & 102.9 \\
\hline 15. Clusters & Epidote & 39.3 & 22.7 & 11.0 & 0.5 & 23.2 & 0.1 & & 0.2 & 0.2 & 97.2 \\
\hline 477A-10,CC, No. 2 & & & & & & & & & & & \\
\hline 1. Ragged grain & Epidote & 38.9 & 27.6 & 6.2 & 0.2 & 23.8 & & & 0.1 & 0.1 & $97.0^{3}$ \\
\hline $\begin{array}{l}\text { 2. Clear, in patch of } \\
\text { sulfide; euhedral }\end{array}$ & Ab98 + & 71.6 & 18.3 & 0.1 & & 0.5 & 12.3 & 0.1 & & & 102.8 \\
\hline 3. Altered plagioclase & Albite & 59.8 & 16.3 & 0.3 & 0.1 & 1.9 & 11.5 & 0.1 & 0.1 & 0.6 & 90.7 \\
\hline 4. Anhedral in sulfide & Quartz & 112 & & & & & & & & & \\
\hline 6. Long, euhedral lath & Abs6-60 & 59.2 & 23.8 & 0.8 & 0.1 & 7.9 & 8.1 & 0.6 & 0.2 & 0.1 & $100.6^{\mathrm{a}}$ \\
\hline 7. Lath, clear & $\mathrm{Ab}_{49-52}$ & 56.0 & 26.3 & 0.6 & 0.1 & 10.5 & 7.0 & 0.3 & 0.1 & 0.1 & $101.1^{\mathrm{a}}$ \\
\hline 8. Small grain & Epidote & 39.9 & 26.7 & 6.8 & 0.3 & 23.7 & & & 0.1 & 0.2 & $97.9^{\mathrm{a}}$ \\
\hline 9. Blue CL plag. & $\mathrm{Ab}_{45}-49$ & 54.6 & 27.3 & 0.7 & 0.1 & 11.8 & 6.0 & 0.3 & 0.1 & 0.1 & 100.9 \\
\hline 10. Small & Epidote & 37.2 & 23.5 & 8.5 & 0.4 & 22.9 & 0.1 & 0.1 & 0.1 & 0.5 & 93.3 \\
\hline 11. Prism & Epidote & 39.7 & 26.8 & 6.7 & 0.3 & 23.8 & & & 0.1 & 0.1 & 97.6 \\
\hline 12. Small, high bf & Epidote & 39.7 & 27.4 & 6.6 & 0.3 & 23.2 & & & 0.1 & 0.1 & 97.4 \\
\hline 13. Plagioclase & $A b_{52-58}$ & 56.6 & 26.2 & 0.2 & & 10.1 & 7.0 & 0.2 & & & 100.2 \\
\hline 14. Tiny euhedral & Sphene & 34.9 & 2.8 & 0.6 & 0.1 & 30.3 & & & 35.2 & & 104.0 \\
\hline
\end{tabular}


Table 2. (Continued).

\begin{tabular}{|c|c|c|c|c|c|c|c|c|c|c|c|}
\hline \multirow{2}{*}{$\begin{array}{l}\text { Measurement } \\
\text { Description }\end{array}$} & \multirow[b]{2}{*}{ Interpretation } & \multicolumn{10}{|c|}{ Composition $(\%)$} \\
\hline & & $\mathrm{SiO}_{2}$ & $\mathrm{Al}_{2} \mathrm{O}_{3}$ & $\mathrm{FeO}$ & $\mathrm{MnO}$ & $\mathrm{CaO}$ & $\mathrm{Na}_{2} \mathrm{O}$ & $\mathrm{K}_{2} \mathrm{O}$ & $\mathrm{TiO}_{2}$ & $\mathrm{MgO}$ & Total \\
\hline \multicolumn{12}{|l|}{$477 \mathrm{~A}-12, \mathrm{CC}$} \\
\hline 1. Center, subhedral & $\mathrm{Ab}_{76}$ & 63.6 & 22.2 & 8.5 & & 5.1 & 8.5 & 0.7 & & & 100.3 \\
\hline 2. Large, cement & Quartz & 113 & & & & & & & & & \\
\hline $\begin{array}{l}\text { 3. Small prisms in } \\
\text { pore space }\end{array}$ & Quartz & 103 & & & & & & & & & \\
\hline 4. Plagioclase & & 61.0 & 24.2 & 0.2 & & 7.2 & 9.3 & 0.3 & & & 102.2 \\
\hline 5. Prism & Epidote & 39.0 & 27.1 & 7.2 & 0.4 & 23.0 & & & 0.1 & 0.4 & $97.0^{\mathrm{a}}$ \\
\hline 6. Center of spray & Epidote & 37.9 & 25.2 & 8.1 & 0.4 & 23.0 & 0.1 & & 0.2 & 0.3 & $95.1^{\mathrm{a}}$ \\
\hline 7. Stubby prism & Epidote & 39.5 & 23.6 & 6.3 & 0.3 & 23.0 & 0.1 & & 0.2 & 0.6 & 93.6 \\
\hline 8. Long prism & Epidote & 37.5 & 23.8 & 9.9 & 0.3 & 23.1 & & & 0.1 & 0.3 & $95.1^{\mathrm{a}}$ \\
\hline $\begin{array}{l}\text { 9. Small grains in } \\
\text { matrix }\end{array}$ & ?Zeolite & 48.3 & 14.7 & 0.1 & & 1.7 & 7.3 & 0.1 & & 0.1 & 72.3 \\
\hline 10. Opaque, ore & Pyrrhotite & 0.6 & & 80.2 & & & & & & & \\
\hline 11. Long prism edge & Epidote & 40.6 & 24.1 & 8.7 & 0.3 & 23.3 & & & 0.2 & 0.1 & $97.4^{\mathrm{a}}$ \\
\hline 12. Homogeneous edge & Epidote & 38.1 & 25.2 & 7.5 & 0.4 & 22.9 & 0.1 & & 0.2 & 0.4 & $95.5^{\mathrm{a}}$ \\
\hline $\begin{array}{l}\text { 13. Homogeneous } \\
\text { middle }\end{array}$ & Epidote & 38.8 & 24.0 & 9.7 & 0.7 & 22.7 & & & 0.9 & 0.2 & $97.1^{\mathrm{a}}$ \\
\hline 14. Homogeneous end & Epidote & 37.0 & 24.3 & 5.9 & 0.4 & 22.7 & & & 0.1 & 0.7 & 91.2 \\
\hline \multicolumn{12}{|c|}{ 477A-11-1, 11-15 cm (R. Oberhansli, operator) } \\
\hline 1. Epidote & & 37.8 & 28.4 & 5.8 & 0.3 & 23.7 & & & 0.2 & 0.4 & 96.4 \\
\hline 2. Epidote & & 38.0 & 27.9 & 6.4 & 0.5 & 23.3 & & & & 0.3 & 96.4 \\
\hline 3. Epidote & & 37.8 & 27.1 & 7.5 & 0.5 & 23.4 & & & 0.1 & 0.2 & 96.6 \\
\hline 4. Epidote & & 37.8 & 26.6 & 8.0 & 0.2 & 23.8 & & & 0.3 & 0.2 & 96.9 \\
\hline 5. Epidote & & 38.2 & 27.6 & 6.8 & 0.2 & 23.8 & & & 0.1 & 0.1 & 96.7 \\
\hline 6. Epidote & & 37.3 & 25.1 & 9.5 & 0.1 & 23.1 & & & 0.2 & 0.7 & 96.0 \\
\hline 7. Epidote, high bf & & 37.3 & 23.8 & 10.7 & 0.3 & 23.7 & & & 0.2 & 0.4 & 96.5 \\
\hline 8. Epidote, fiber & & 37.7 & 25.7 & 8.7 & 0.2 & 23.6 & & & 0.3 & 0.2 & 96.5 \\
\hline $\begin{array}{l}\text { 9. Epidote, small, } \\
\text { high bf }\end{array}$ & & 38.3 & 25.9 & 7.8 & 0.5 & 23.0 & & & 0.2 & 0.2 & 95.5 \\
\hline 10. Epidote, matrix & & 37.8 & 27.1 & 7.4 & 0.3 & 23.6 & & & 0.3 & 0.2 & 96.7 \\
\hline 11. Epidote & & 37.4 & 25.5 & 8.8 & 0.2 & 23.2 & & & 0.1 & 0.5 & 95.7 \\
\hline 12. Plagioclase, twinned & Ab56 & 57.3 & 26.4 & 0.2 & & 8.3 & 6.2 & 0.2 & 0 & & 98.7 \\
\hline 13. Plagioclase & Abs5 & 57.8 & 26.1 & 0.1 & & 7.7 & 6.6 & 0.4 & 0.1 & & 98.7 \\
\hline 14. Plagioclase & $\mathrm{Ab}_{57}$ & 58.9 & 25.2 & 0.1 & 0.1 & 6.9 & 7.0 & 0.7 & & & 98.9 \\
\hline 15. Plagioclase & Abs9 & 59.6 & 25.4 & 0.2 & 0.1 & 7.0 & 6.6 & 0.7 & & & 99.5 \\
\hline 16. Plagioclase & $\mathrm{Ab}_{60}$ & 61.5 & 24.6 & 0.1 & & 5.8 & 6.8 & & & & 98.8 \\
\hline 17. Plagioclase & A $060-65$ & 60.3 & 25.3 & 0.1 & & 6.5 & 7.5 & & & & 99.7 \\
\hline 18. Plagioclase & $\mathrm{Ab} 48$ & 56.4 & 27,4 & 0.4 & 0.1 & 9.2 & 5.4 & 0.6 & 0.1 & & 99.5 \\
\hline $\begin{array}{l}\text { 19. Plagioclase, } \\
\text { euhedral, small }\end{array}$ & Albite & 67.7 & 21.4 & & & 1.4 & 9.7 & & & & 100.2 \\
\hline 20. Plagioclase & $\mathrm{Ab}_{45}$ & 54.0 & 27.4 & 0.7 & 0.1 & 10.8 & 4.5 & 0.5 & 0.1 & & 98.2 \\
\hline 21. Plagioclase & Albite & 67.0 & 21.5 & & & 1.5 & 5.4 & 0.5 & 0.3 & 0 & 96.2 \\
\hline 22. Matrix & Chlorite? & 22.4 & 14.4 & 13.0 & 0.4 & 0.1 & 0.2 & 0.1 & & 17.5 & 67.9 \\
\hline 23. Unknown & ?Prehnite & 57.8 & 17.0 & 7.2 & 0.0 & 15.7 & & & 0.1 & 0.3 & 98.0 \\
\hline 24. Unknown & Mixture? & 86.4 & 6.5 & 0.4 & & 1.2 & 1.6 & & 0.4 & & 96.4 \\
\hline 25. Matrix?? & & 43.4 & 26.8 & 5.7 & 0.2 & 20.1 & 1.8 & & & & 97.8 \\
\hline 26. Unknown & ?Zeolite? & 46.8 & 24.6 & 3.3 & & 1.3 & 2.2 & 7.4 & 0.1 & 1.1 & 86.8 \\
\hline $\begin{array}{l}\text { 28. Pyrrhotite, } \\
\text { intergranular }\end{array}$ & & 0.5 & & $79.5^{\mathrm{b}}$ & & & & & & . & 年 \\
\hline 29. Pyrrhotite, rod & & 0.4 & & $79.3^{\mathrm{b}}$ & & & & & & & \\
\hline 30. Pyrrhotite, large & & 0.2 & 0.3 & $79.8^{b}$ & & & & & & & \\
\hline 31. Pyrrhotite & & 0.5 & 0.2 & $79.1^{\mathrm{b}}$ & & & & & & & \\
\hline 32. Pyrrhotite & & 1.4 & 0.3 & $76.4^{b}$ & & & & & & & \\
\hline 33. Pyrrhotite, cluster & & 0.4 & 0.4 & $79.6^{\mathrm{b}}$ & & & & & & & \\
\hline
\end{tabular}

478-22,CC (Sandstone)

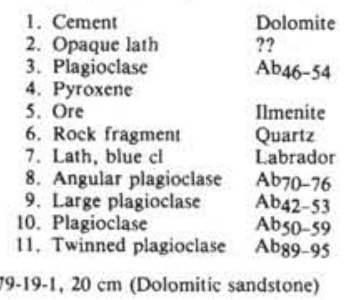

99-19-1, $20 \mathrm{~cm}$ (Dolomitic sandstone)

1. Plagioclase, fresh Abs8-70

Absed

. Plagioclase, angular $\mathrm{Ab}_{44-53}$

477 A-5-1 (M. Lyle, operator)

1. Epidote

3. Epidote

Pyrrhotite

5. Pyrrhotite
6. Pyrrhotite

( $\left.\mathrm{Fe}_{0.9} \mathrm{Mn}_{0.003} \mathrm{~S}\right)$

477-22-2 (M. Lyle, operator)

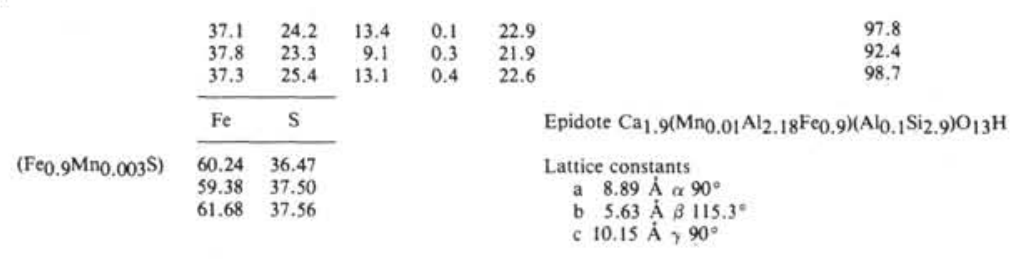

$\begin{array}{llll}\left(\mathrm{Fe}_{0.99} \mathrm{Mn}_{0.01} \mathrm{~S}_{2}\right) & 46.59 & 53.91\end{array}$

Note: $\mathrm{cl}=$ cathode luminescence, $\mathrm{bf}=$ birefringence.

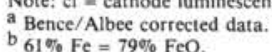


(Plate 1). Possibly part of the lithification of low-permeability brittle claystones (as found in median cores, as, for example, Core 477-17 to Core 477-22) reflects quartz cementation.

\section{Feldspars}

As previously mentioned, silt- to sand-sized plagioclase and some $\mathrm{K}$-feldspar persist as relict grains to the bottom of the hole. Many are clouded with microlitic alteration products (Plate 1). Clear centers on plagioclase show strong blue cathode luminescence and median compositions similar to the detrital suite (Table 2)-traits considered diagnostic of relict status. In bulk X-ray diffraction samples, K-feldspar abundance does not seem to vary much until about 180 meters, below which grains are no longer detected. Niemitz (this volume, Pt. 2) reports a uniform $\mathrm{K}_{2} \mathrm{O}$ content in bulk powder samples to a depth of 190 meters, below which it trails off quickly. Evidence for neoformation of K-feldspars derives from smear slide and SEM observations on the finest grain sizes, where they occur as $4-10 \mu$ m pinacoids in Cores 477-16 to 477-18. Microprobe analyses from Core 477-17 (Table 2) revealed pure K-feldspar as overgrowth and as euhedral small crystals.

Albite was detected in the uppermost microprobe sample (Core $477-17 ; 123 \mathrm{~m}$ ) as 10-15 $\mu$ m euhedral crystals showing no, or faint, cathode luminescence. Although not abundant, it occurs in all lower samples as thin overgrowths on a variety of grains or as small crystals. A high-temperature origin for newly formed albite might be indicated by a consistently slight calcium content of 1 to $4 \%$.

\section{Clay Minerals}

Below the sill, the proportional content of clay minerals also increases. There is a change from the detrital spectrum of illite-smectite-mixed-layer-chlorite-kaolinite above. Smectite and mixed-layer clay dominate Core $477-15(\sim 110 \mathrm{~m})$ but illite increases in abundance at about 119 meters and persists to Core 477-21 ( $163 \mathrm{~m})$. Chlorite also becomes prominent at about 120 meters, increasing to 155 meters, below which it is the exclusive clay mineral until 249 meters. The composition of chlorite shifts some in upper cores, but below Core 477-22 it shows very pronounced, crisp symmetric peaks indicating a $\mathrm{Mg} / \mathrm{Fe}$ ratio of about 1.34 (Fig. 3). Microprobe values, although rather qualitative, suggest a trace of manganese and $\mathrm{Mg} / \mathrm{Fe}$ ratios around 1.0 to 1.9. The chlorite has very low birefringence and exhibits extremely regular, smooth-surfaced, interpenetrating euhedral platelets forming a porous card-house structure (Plate 3). Rather than a cement, much of the chlorite appears as part of the microlitic replacement of detrital grains. Abundant clay-sized opaque prisms are also associated with the chlorite matrix.

The lowest sample examined (477A-11-1, $15 \mathrm{~cm}$ ) represents an abrupt departure from the chlorite spectrum above, and deserves further attention. Microtextures show thin, irregular, curved, onlapping sheaves. In X- ray patterns, clay mineral content decreases and chlorite peaks are replaced by a broad asymmetric peak from 12.8 to $14.2 \AA$, perhaps indicating neoformation of a chlorite-mixed-layer phase. Porosity remains high.

\section{Carbonates}

Our understanding of the relation of carbonate minerals to the hydrothermal assemblage is still tenuous. Micritic dolomite giving high isotopic temperatures of formation $\left(100^{\circ} \mathrm{C}+\right)$ occurs as hard rocks in the first cores beneath both sill horizons (Kelts and McKenzie, this volume, Pt. 2). These rocks may, however, have formed as a contact mineral, like dolomite occurrence near other sills (see Kastner, this volume, Pt. 2). In addition, dolomite is also detected as a diagenetic mineral in the unaltered anoxic oozes of Core 477-5. The dolomite sample at the top of Core 477-19 is strongly suspected to derive from downhole cavings of diagenetic dolomite, because of the presence of trapped diatom frustules and dolomite-replaced, but recognizable miliolid foraminifers. On the other hand, dolomite an chiometric magnesian carbonates are detected in bulk X-ray patterns from claystones from Cores $477-15$ to 477-17, and these seem related to the hydrothermal gradient. From Core 477-19 to 477-23 ( 145-190 m), stoichiometric calcite reappears as small micrite crystal aggregates within black pyritic mudstones. Below this level carbonate is rare but was occasionally detected optically as traces of a very high birefringent phase (siderite?) encompassing sulfides (Fig. 5, later).

\section{Sulfates-Sulfides}

Pore-water sulfate content is reduced to almost zero within a few meters sub-bottom in the anoxic diatom oozes (Gieskes et al., this volume, Pt. 2), and small pyrite framboids are abundant as grains within diatom frustules. We were thus surprised to encounter abundant anhydrite and some associated gypsum in medium gray mudstones beneath the sill at Hole 477. Anhydrite occurs as clotted, white clusters of fine-grained $(20-60 \mu \mathrm{m})$ equidimensional smooth crystals (Plate 2) and as disseminations in light gray laminae. Except in the clusters which are dispersed sporadically in low-permeable mudstones from 106 to 128 meters (Cores $477-15$ to $477-17$ ), anhydrite is not a major component of bulk samples.

Small amounts of pyrite are also present from Cores 477-15 to $477-18$, but pyrite, and possibly some other sulfides, become very abundant in dark gray, brittle mudstones in the interval from Core 477-19 to 477-23. In some cores, flushing with drill fluids has concentrated large pyrite crystals (up to $5 \mathrm{~mm}$ ) with characteristic cubic habit and bulging striated surfaces. Pyrite is less abundant below this level, but persists as small cubic crystals as for example in Core 477A-5 to Core 477A-10 (Fig. 5).

Pyrrhotite was positively identified in Core 477-22 (Fig. 4) and is common to the bottom of the hole. It occurs as long subhedral to euhedral prisms, and small ( 20 $\mu \mathrm{m}$ ) hexagonal platelets (Fig. 5). Pyrrhotite is common- 


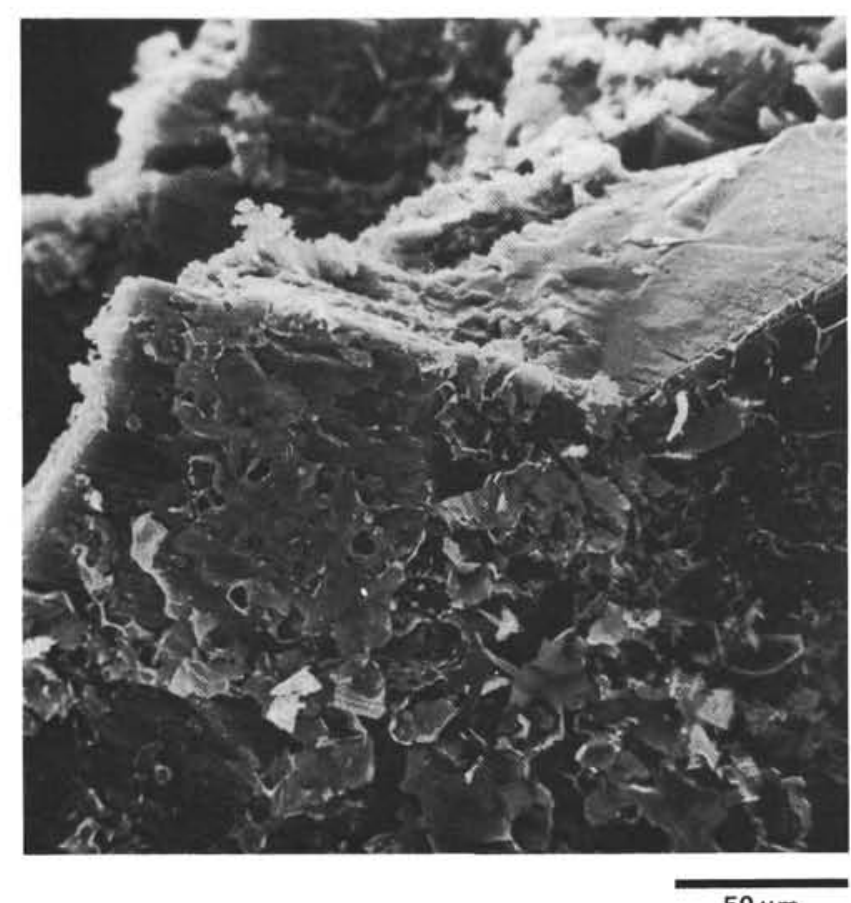

$50 \mu \mathrm{m}$

Figure 4. Scanning electron microphotograph of the oxidized surface of a pyrrhotite crystal isolated by ultrasonic dispersion from sandstone Sample $477-22-2,35 \mathrm{~cm}$.

ly present in patches as a late stage pore-filling cement in sandstone samples. It has a nearly stoichiometric composition with a trace of manganese (Table 2).

Evidence from cursory reflected light microscopy, $\mathrm{XRD}$, and spot microprobe measurements on opaque minerals suggests that other ore sulfides are probably present in some samples. McKibben (1979) studied the sulfide minerals in the Imperial Valley geothermal fields and found similar zones of large, euhedral porphyro- blasts of pyrite as well as galena, sphalerite, and chalcopyrite occurring as cements below the pyrite level.

\section{Zeolite}

Some clinoptilolite occurs in hard mudstones just above the sill and a trace of sodium zeolite (analcime?) was encountered in Sample 477A-6,CC. Zeolites are not conspicuous below the sill, but their presence is inferred from a few smear slides and SEM photographs which show a high alumina, iron-rich fibrous phase, which may also be talc.

\section{Epidote Group}

The first traces of epidote encountered are tiny, slender needles in Core 477-19 siltstone. Epidote is common below Core 477-23. Various types of epidote crystal habits may coexist within the same sample, including blocky small crystals, long slender prisms, and elongate, doubly terminated fat prisms (Plate 1). Small prisms replace feldspar grains, but large euhedral grains commonly occur as clusters in interstitial spaces. Small $(\sim 10-50 \mu \mathrm{m})$ birefringent grains, originally identified as clinozoisite, were proved by microprobe measurements to be epidote. Analyses showed that all varieties of epidote have an iron ( $\mathrm{FeO}$ ) content of 6.4 to $12.0 \mathrm{wt} . \%$ and lack cathode luminescence. There is no clear downhole chemical trend. The larger crystals have generally higher iron content, and these are more abundant in sandstone Samples 477A-5,CC and 477A-10,CC, in which epidote constitutes up to $15 \%$ of the rock.

\section{Accessories}

Sphene was positively identified at 240 meters (Core $477 \mathrm{~A}-10$ ) and optically recognized in all samples below 200 meters as a trace mineral $(<10 \mu \mathrm{m})$, which is conspicuous because of its high dispersion. In the lowermost sample (Sample 477A-11-1, 11-15 cm) there is

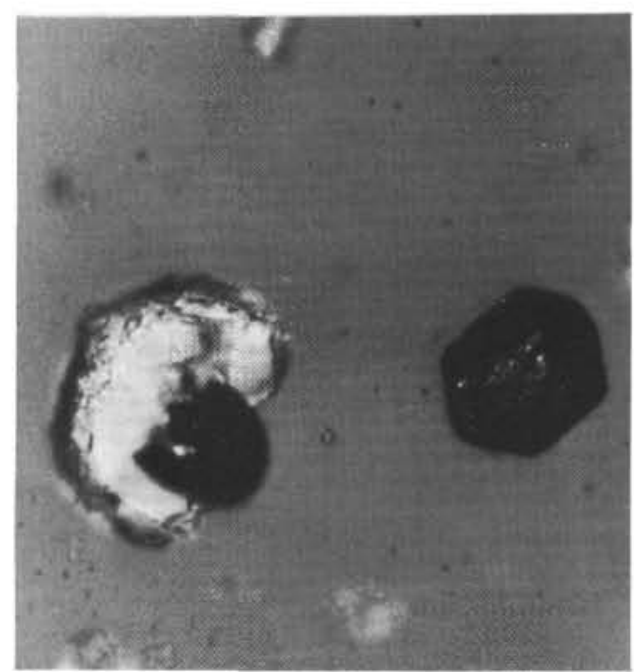

A

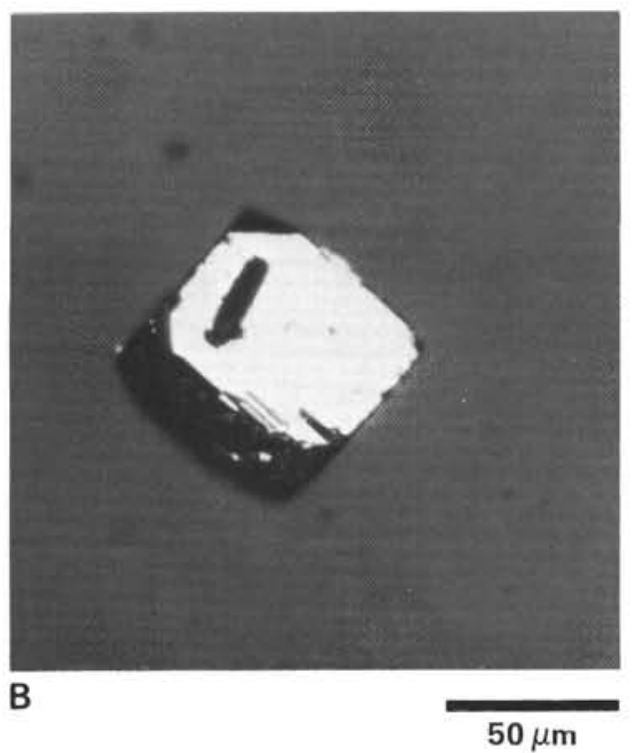

$50 \mu \mathrm{m}$

Figure 5. Reflected light microphotograph of sulfides sieved from Sample 477A-10-1, 28-30 cm. A. Hexagonal (pyrrhotite?) platelet and another encased in a carbonate grain with serrated edges. B. Pyrite cube. 
some XRD evidence for fine-grained prehnite, but the identification is tentative. Carbon coatings are present on many of the $<30 \mu \mathrm{m}$ grains, which makes optical identifications quite difficult. Sandstone samples from Hole 477A all contain bits of relict wood and other organic matter which have been transformed to the presence of hard black coal.

\section{Zonation}

Although the foregoing mineralogical data remain incomplete, they clearly indicate the presence of zones of progressively higher grade metamorphism to lower greenschist facies that are consistent with a large, deep-seated heat source producing a very steep thermal gradient. What follows is a summary of characteristic authigenic parageneses, with a note of caution that the depth boundaries are not fixed, and indeed are expected to change with further petrology.

1) 0-50 meters, Hole 477. Anoxic, diatomaceous muds and silty sands without significant alteration. Sands rich in volcanic plagioclase, quartz and up to $20 \%$ detrital clay minerals including illite, chlorite, and smectite with traces of kaolinite. Up to $50 \%$ of the highly porous sediment consists of reactive opaline silica frustules associated with $2-4 \%$ organic carbon and $1 \%$ framboidal pyrite. Compaction effects related to emplacement and traces of clinoptilolite occur near the upper sill (Einsele et al., 1980).

2) $105-125$ meters, Cores $477-15$ to $477-17$. Anhydrite-dolomite claystones with color changes from brown to gray as illitic clay is replaced by new chlorite.

3) 125-140 meters, Cores 477-16-477-18. Illite-chlorite-pyrite-quartz claystones, with possible $\mathrm{K}$-feldspar and zeolite (poor recovery over this interval).

4) 140-190 meters, Cores 477-19-477-22. Chlorite-pyrite-quartz-calcite-carbonaceous claystones with traces of K-feldspar, albite, and epidote. Calcite minor and pyrite abundant as mm-sized cubes. Traces of pyrrhotite.

5) 190-260 meters, Cores $477-23$ and $477 \mathrm{~A}-5-11$. Chlorite-quartz-epidote-albite-pyrrhotite-sphene sandstone. Includes many relict mineral grains. Evidence for the formation of new phyllosilicates near the bottom of the section.

Similarly zoned mineral assemblages have been reported from active geothermal fields in continental settings where hydrothermal systems are reacting with thick piles of sediment (e.g., Cesano, Italy-Baldi et al., 1976; or Imperial Valley, U.S.A., and Cerro Prieto, Mexico-McDowell and Elders, 1979, and Hoagland and Elders, 1978). Comparisons of Site 477 with the isotherm boundaries of these fields would suggest that the assemblage at the base of Hole 477A represents temperatures just under $300^{\circ} \mathrm{C}$ (Elders et al., 1979). Core 477-16, where dolomite disappears, should represent an approximately $200^{\circ} \mathrm{C}$ isotherm. Even allowing for the uncertainties, it is conspicuous that the change of parageneses within 100 meters in Holes 477-477A corresponds to the change that is observed in traversing a vertical distance of 400 meters or more in the Cerro Pri- eto Field. This illustrates the particularly steep gradient in the Guaymas Basin site. Temperature comparisons are only qualitative, and in fact there are numerous petrological or geochemical differences between the oceanic and continental systems. For example, the Salton Trough system has a thick carbonate (calcite) cap, and neoformation of dolomite is not described in the anhydrite zone. Comparisons with continental geothermal fields must take into account several peculiarities of the Site 477 system: (1) Fluids are only slightly above seawater ion concentrations (Gieskes et al., this volume, Pt. 2) rather than concentrated brines which are the rule in continental geothermal fields. (2) Recharge of the Site 477 system must derive from a sea-water reservoir, without meteoric input. If there is a steam envelope, a deep ocean hydrothermal convection cell will have an added 2000-meter hydrostatic head. (3) The original sediment of Site 477 has a different composition, with far less of the potassium-bearing phases and with an abundance of reactive opaline silica. Temperatures of formation estimated from present isotherms in continental geothermal areas could have been slightly underestimated because of cooling effects of drill fluids (McDowell and Elders, 1979). (4) $p_{\mathrm{CO}_{2}}$ is an important parameter for mineral equilibria, but was not determined at Site 477.

In many aspects the Cerro Prieto Field seems most similar to Holes 477 and 477A and provides a useful analogy for interpreting textures and mineralogy. In contrast, the Cesano Field in Italy (Baldi et al., 1976) is much more dominated by secondary carbonates because hydrothermal fluids are interacting with limestones. There are, however, also zones of anhydrite "microgeodes" and abundant quartz in prismatic habit. Samples from the Cerro Prieto Field commonly show some zones of similarly high porosities $(15-30 \%)$ in sandstones which show extensive alteration (Lyons and van de Camp, 1980). The removal of unstable grains and diatom frustules from Site 477 has apparently just kept pace with the formation of new minerals occurring either as interstitial growth or microlitic replacements. Comparison of porosity, epidote crystal habit, and mineral assemblage suggests that Cores $477 \mathrm{~A}-5$ through 477A-10 correspond to the base of the "porous zone" defined by McDowell and Elders (1979), or about midway into a calc-silicate zone representing $270-300^{\circ} \mathrm{C}$ (Elders et al., 1979). Site 477 and the Cerro Prieto Field also share the following features: the dominant minerals are quartz and chlorite, whereas neofeldspars, zeolites, sphene, and pyrrhotite are generally present in trace amounts; relict mineral grains are abundant, commonly with surface replacement and overgrowth.

Examination of several features also suggests that the intrusion is young and induces the speculation that the site might actually be in the process of heating up:

1) If we accept the estimates of $70^{\circ} \mathrm{C}$ (on the basis of anhydrite) for the base of the sill and $270^{\circ} \mathrm{C}$ for the base of the hole (as conservatively estimated from mineral evidence), a gradient of $200^{\circ} \mathrm{C} / 100 \mathrm{~m}$ results. This would imply a magma source only a few hundred meters below. 
2) Relict minerals with thin overgrowths are abundant, and texture relations between acicular and blocky epidotes suggest that the latter, which represent warmer conditions, are the latest-stage precipitate.

3) Lack of hornfels texture or pervasive quartz cement, in spite of the abundance of opal, suggests that abundant silica is being transported away.

4) Heat flow pattern anomalies show steep flank gradients (Williams et al., 1979) at Site 477.

5) Recent submersible observations show active fumaroles near this site (Gieskes et al., this volume).

The presence of anhydrite is curious. Hoagland and Elders (1978) have interpreted the appearance of anhydrite at Cerro Prieto in terms of recharge: it would form along with carbonate at sites of descending cold water. A locus for recharge is not yet known in the Guaymas Basin although faults are abundant, and the dolerite sills shows a surprisingly high porosity (see Site 477 site chapter, this volume, Pt. 1). Thus recharge may have been associated with the sill intrusion. Anhydrite has been a typical quench product of sea-water-basalt reaction experiments conducted with high water/rock ratios (Seyfried and Bischoff, 1979). These experiments do not include sulfate reduction, which occurs rapidly in Guaymas Basin muds. The mode in which anhydrite occurs at Site 477 seems consistent with a quenching origin, but further evidence is needed.

\section{CONCLUSIONS}

At Site 477, we recovered, from shallow depths below an upper cold sill, rocks which document a steep gradient of progressive hydrothermal alteration of hemipelagic and turbiditic sediments down to a mineral paragenesis approaching $300^{\circ} \mathrm{C}$. Beds show differential lithification and porosity, partly inherited from original lithologies, which probably have also influenced levels of alteration. Although the mineral assemblage at depth is equivalent to lower greenschist facies metamorphism, strictly speaking the rocks are not identical. At Site 477, high porosity, abundant reactive silica, low overburden, and apparently high water/rock ratios combine to produce characteristically euhedral hydrothermal minerals. Rocks show numerous similarities with counterparts in active geothermal areas of the Salton Sea region, particularly Cerro Prieto.

Clearly Site 477 requires further detailed study, particularly to identify accessory and metallic phases and to separate hydrothermal from relict phases. It provides a unique opportunity as an in situ experiment for comparison of equilibrium models developed for geothermal systems. A comparison of independent geothermometers, for example vitrinite reflectance of coaly fragments, is needed. Criteria needed to recognize this type of early oceanic metamorphism of sediments in ancient geologic formations might include idiomorphic habits cemented by late-stage quartz, abundant relict mineral cores, and steep mineral gradients.

\section{ACKNOWLEDGMENTS}

I appreciate long discussions with Jeffrey Niemitz, Gerhard Einsele, Mitchell Lyle, Miriam Kastner, and Joris Gieskes on board the Glomar Challenger while puzzling over these unusual rocks. H. U. Franz helped with SEM, U. Gerber with photographs, R. Oberhansli and C. Honegger with the microprobe. I thank the DSDP art department for their work on the illustrations. This note was written while I was a guest of the Dépt. de géotectonique, Université Pierre et Marie Curie, Paris, on a Swiss Nationalfond fellowship. I gladly acknowledge the particularly thoughtful reviews of T. Barrett and K. Hsü.

\section{REFERENCES}

Baldi, P., Civitelli, G., Funiciello, R., Lombardi, G., Purotto, M., and Serva, L., 1976. Study of the stratigraphy and mineralization of the deep wells in Cesano Geothermal Field. Proc. Int. Congr. Therm. Waters Geotherm. Energy Vulcanism Mediterr. Area. (Vol. 1): Athens, Oct., 1976, 71-87.

Bischoff, J. L., 1969. Red Sea geothermal brine deposits: Their mineralogy, chemistry and genesis. In Degens, E. T., and Ross, D. A. (Eds.), Hot Brines and Recent Heavy Metal Deposits in the Red Sea: New York (Springer-Verlag), pp. 368-401.

Corliss, J. B., Dymon, J. R., Gordon, L. I., Edmond, J. M., Von Herzen, R. P., Ballard, R. D, Green, K., Williams, D. L., Bainbridge, A., Crane, K., and Van Andel, Tj. H., 1979. Submarine thermal springs on the Galapagos Rift. Science, 203:1073-1083.

Einsele, G., Gieskes, J., Curray, J., Moore, D., Aguayo, E., Aubry, M.-P., Fornari, D., Guerrero, J., Kastner, M., Kelts, K., Lyle, M., Matoba, M., Molina-Cruz, A., Niemitz, J., Rueda, J., Saunders, A., Schrader, H., Simoneit, B., and Vacquier, V., 1980. Intrusion of basaltic sills into highly porous sediments, and resulting hydrothermal activity. Nature, 283:441-445.

Elders, W. A., Hoagland, J. R., McDowell, S. D., and Cobo, J. M., 1979. Hydrothermal mineral zones in the geothermal reservoir of Cerro Prieto. Guide Book, Field Trip 7: University of California, Riverside, UCR/IGPP-79/23, pp. 36-43.

Francheteau, J., Needham, H. D., Choukroune, P., Juteau, T., Seguret, M., Ballard, R. D., Fox, J. P., Normark, W., Carranza, A., Cordoba, D., Guerrero, J., Rangin, C., Bougault, H., Cambon, P., and Hekinian, R., 1979. Massive deep-sea sulphide ore deposits discovered on the East Pacific Rise. Nature, 277:523-528.

Hoagland, J. R., and Elders, W. A., 1978. Hydrothermal mineralogy and isotopic geochemistry in the Cerro Prieto geothermal field, Mexico. I. Hydrothermal mineral zonation. Geol. Resourc. Counc. Trans., 2:283-286.

Lyons, D. J., and van de Kamp, P. C., 1980. Subsurface Geological Geophysical Study of the Cerro Prieto Geothermal Field, Baja California, Mexico. Lawrence Berkeley Laboratory Rpt. Series 10540, Cerro Prieto II, UC66b.

McDowell, S. D., and Elders, W. A., 1979. Geothermal metamorphism of sandstone in the Salton Sea geothermal system. Guide Book, Field Trip 7: University of California, Riverside, UCR/ IGPP-79/23, pp. 70-76.

McKibben, M. A., 1979. Sulfide minerals in the Salton Sea geothermal system, Imperial Valley, California. Geol. Soc. Am. Ann. Mtg. Abstr. 11/2, p. 91.

Seyfried, W., and Bischoff, J. L., 1979. Low temperature basalt alteration by seawater: An experimental study at $70^{\circ}$ and $150^{\circ} \mathrm{C}$. Geochim. Cosmochim. Acta, 43:1937-1947.

Spiess, F. N., MacDonald, K. C., Atwater, T., Ballard, R., Carranza, A., Cordoba, D., Cox, C., Diaz-Garcia, V., Francheteau, J., Guerrero, J., Hawkins, J., Hagman, R., Hessler, R., Juteau, T., Kastner, M., Larson, R., Luyendyk, B., Macdougall, J., Miller, S., Normark, W., Orcutt, J., and Rangin, C., 1980. Hot springs and geophysical experiments on the East Pacific Rise. Science, 207:1421-1433.

Williams, D. L., Becker, K., Lawver, L. A., and Von Herzen, R. P., 1979. Heat flow at the spreading centers of the Guaymas Basin, Gulf of California. J. Geophys. Res., 84:6757-6769. 

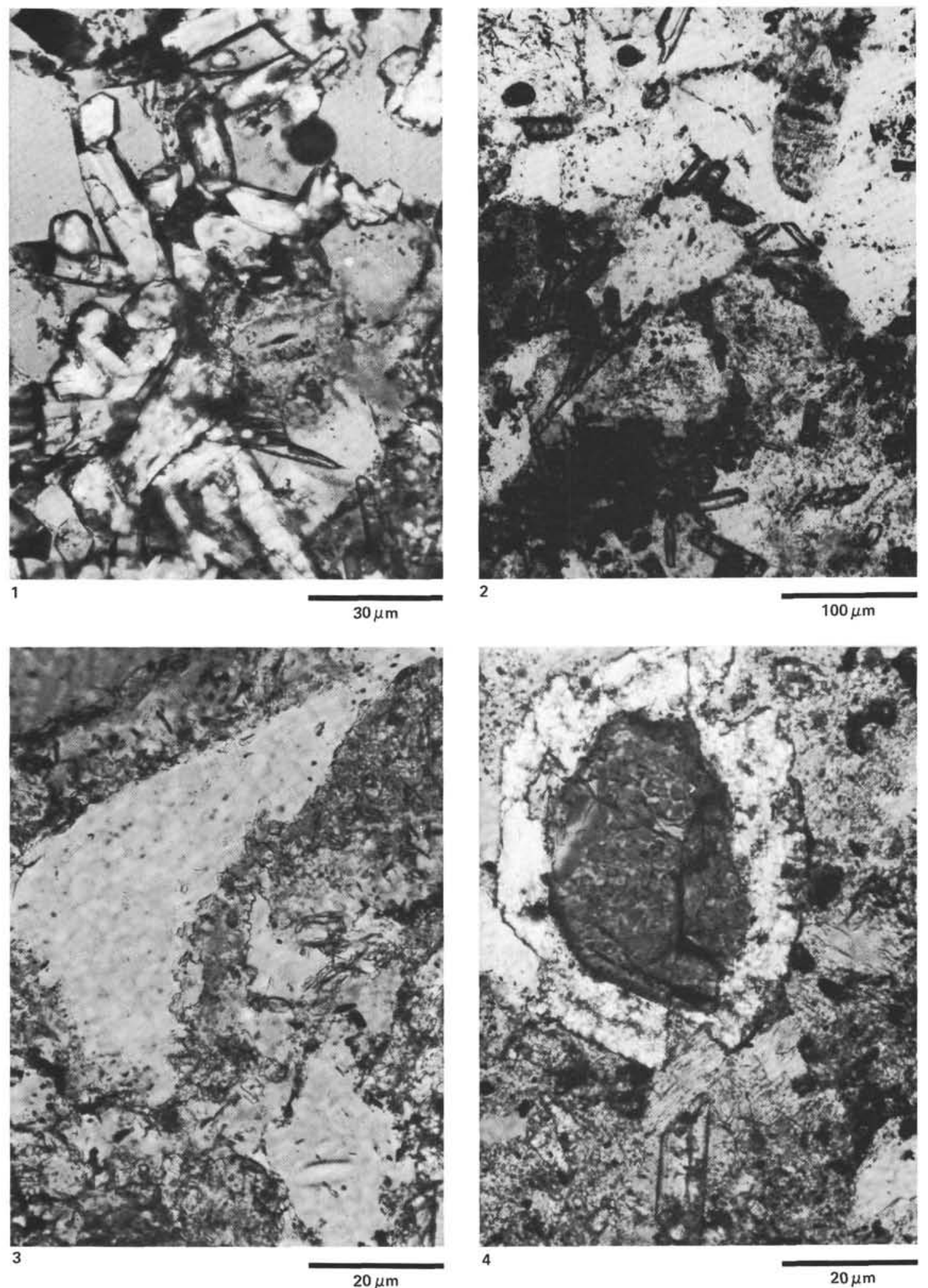

Plate 1. Examples of thin-section microphotographs from hydrothermally altered silty muds. 1. Sample 477A-5-1, $6 \mathrm{~cm}$, a coarse-grained, light gray sandstone: $10-25 \%$ epidote prisms lining relict grains, as clusters or sprays, mostly a high-birefringent variety; $40 \%$ quartz as relict grains and fine replacement mosaics with euhedral to pseudomorph rims (clear patches); $15 \%$ highly altered microlitic plagioclase (clouded). 2 . Sample $477 \mathrm{~A}-5, \mathrm{CC}$, dark gray siltstone with strong lineation: $50 \%$ opaques which are carbon-coated particles; $20 \%$ quartz with euhedral overgrowths; $20 \%$ feldspars. Microphotograph show clusters of large doubly terminated epidote crystals mostly occurring interstitially; both stubby and long acicular prisms are prominent. 3. Sample 477A-10,CC, fine silty-sandstone with small epidotes and serrated relict grains along a quartz mosaic. 4. Sample 477A-11-1, $15 \mathrm{~cm}$, light gray sandstone: detail of an altered, relict plagioclase with a high-birefringent reaction rim and a core replaced by quartz. Penetrated by a long epidote prism. Matrix is fine-grained clay. 

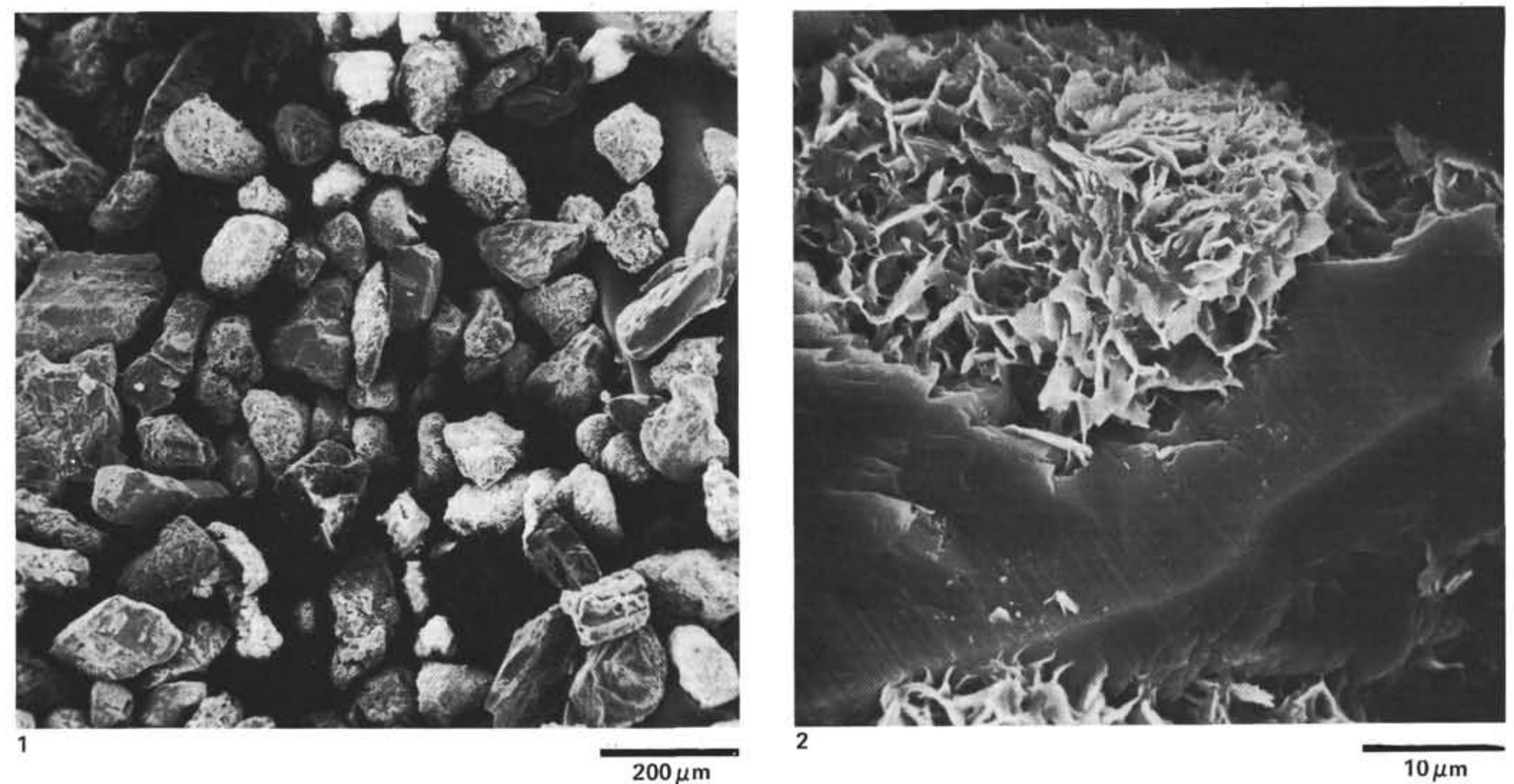

2

$10 \mu \mathrm{m}$
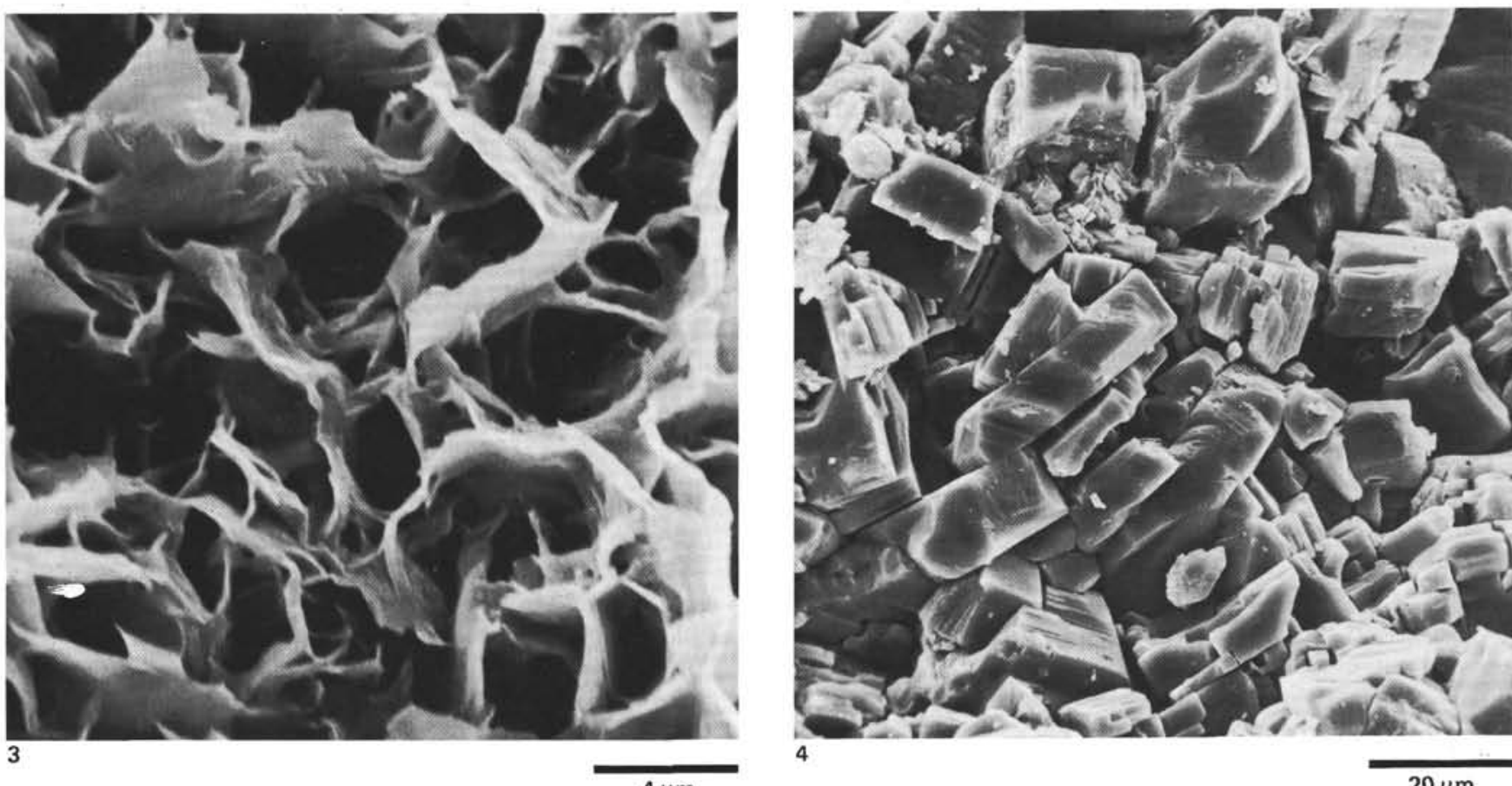

$20 \mu \mathrm{m}$

Plate 2. Scanning electron microphotographs of sand grains sieved from a basal turbidite in unconsolidated diatomaceous muds above the sill. 1-3. Sample 477-7-2, $60 \mathrm{~cm}$, light gray sand, ultrasonically washed and sieved, which shows rounded and etched weathered surfaces of various grains including abundant polygenetic plagioclase, K-feldspar, and quartz, (2-3) note smectite clays adhering to a surface. 4 . Anhydrite cluster from a white clot in the gray mudstone Sample 477-16-3, $20 \mathrm{~cm}$ : equigranular, blocky, subidiomorphic crystals have very smooth surfaces, even at $20,000 \times$ magnification. 

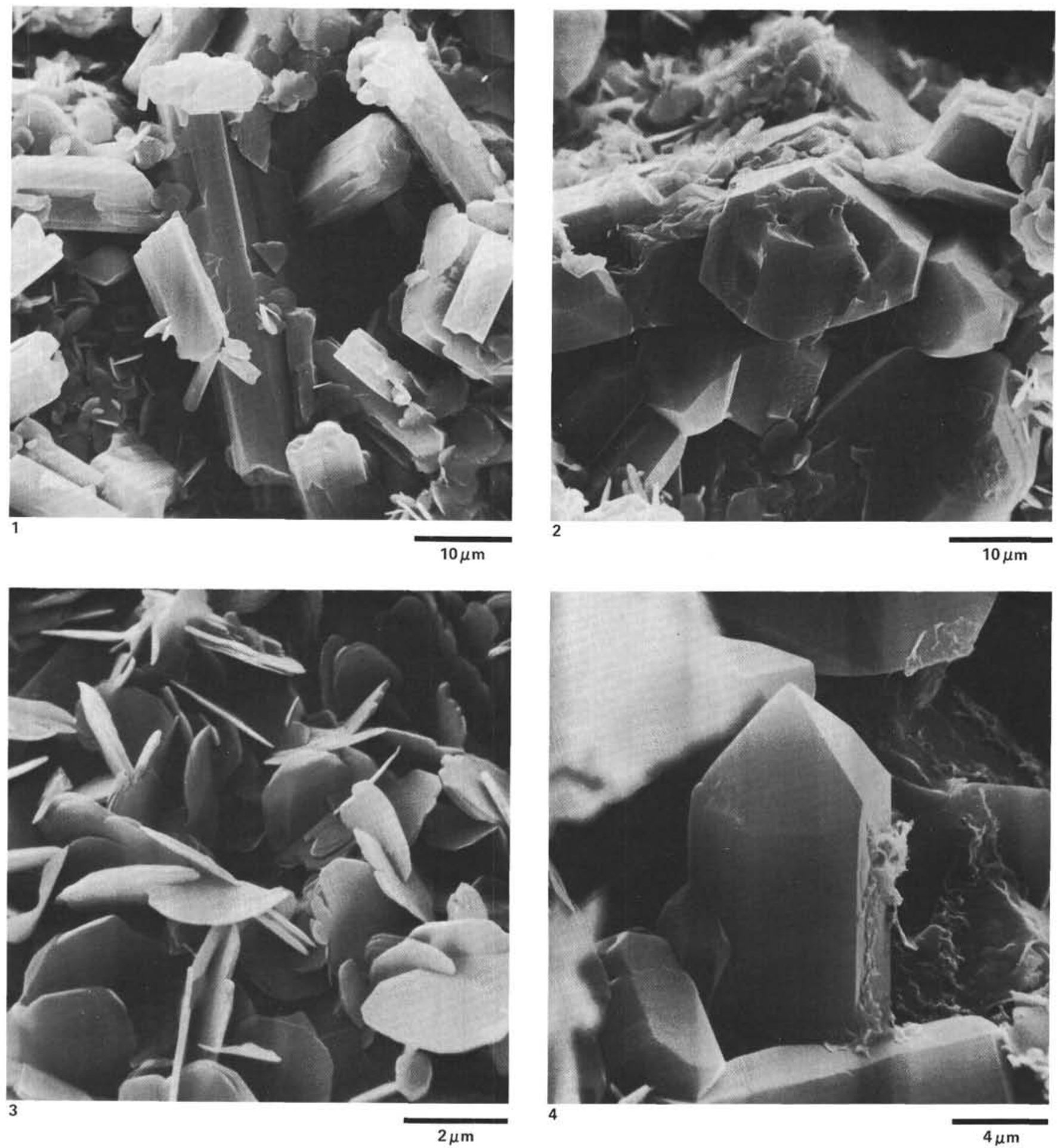

Plate 3. Scanning electron microphotographs of ultrastructure of hydrothermally metamorphosed sandstone. 1. Sample $477-22-2,35 \mathrm{~cm}$, chip of friable light gray sandy-siltstone within dark gray silty clay: broken surface shows concentration of euhedral chlorite flakes and epidote prisms as well as other small blocky crystals (feldspars?) (note apparent porosity). 2-4. Sample 477-10,CC, light gray sandstone: $5 \%$ blocky epidote prisms, $60 \%$ chlorite platelets, and $25 \%$ quartz, (3) well-developed, interlocking Fe-Mg chlorite platelets, (4) euhedral quartz prism termination. 

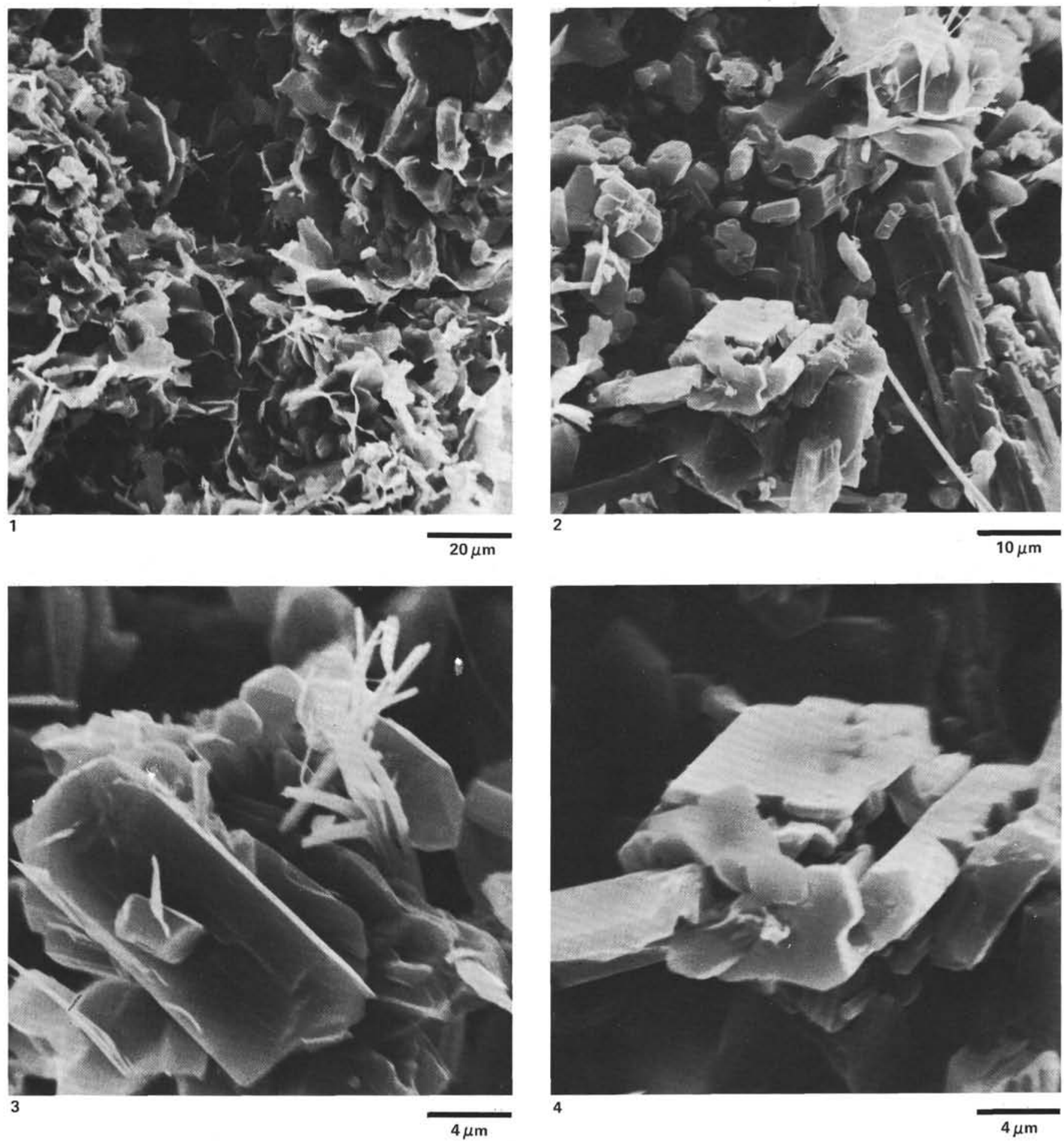

Plate 4. Scanning electron microphotographs of lowermost sample of hydrothermal sediment: Sample 477A-11-1, 15 cm; broken surface. 1. Texture of larger relict grains altered to microlitic mixture; overviews show these are part of coherent grain boundaries, initially $40-60 \mu \mathrm{m}$ large (note porosity and compare phyllosilicate flakes with chlorite in Plate 3). 2. Fine-grained epidote prisms, platelets, and possible tiny albite(?) authigenic pinacoids. 3. Detail of epidote with fibrous mineral of high alumina/silica content, tentatively identified as a zeolite. 4 . Detail of epidote occurring as incomplete grain replacement. 